\title{
Topological invariants of band insulators derived from the local-orbital based embedding potential
}

\author{
H. Ishida, ${ }^{1}$ A. Liebsch, ${ }^{2}$ and D. Wortmann ${ }^{2}$ \\ ${ }^{1}$ College of Humanities and Sciences, Nihon University, Tokyo 156-8550, Japan \\ ${ }^{2}$ Peter Grünberg Institut and Institute for Advanced Simulations, Forschungszentrum Jülich, 52425 Jülich, Germany
}

(Received 27 April 2017; published 11 September 2017)

\begin{abstract}
We demonstrate that topological invariants of band insulators can be derived efficiently from the eigenvalues of the local-orbital (LO) based embedding potential, called also the contact (lead) self-energy. The LO based embedding potential is a bulk quantity. Given the tight-binding Hamiltonian describing the bulk valence and conduction bands, it is constructed straightforwardly from the bulk wave functions satisfying the generalized Bloch condition. When the one-electron energy $\varepsilon$ is located within a projected bulk band gap at a given planar wave vector $\mathbf{k}$, the embedding potential becomes Hermitian. Its real eigenvalues exhibit distinctly different behavior depending on the topological properties of the valence bands, thus enabling unambiguous identification of bulk topological invariants. We consider the Bernevig-Hughes-Zhang model as an example of a time-reversal invariant topological insulator and tin telluride ( $\mathrm{SnTe}$ ) crystallized in a rock-salt structure as an example of a topological crystalline insulator.
\end{abstract}

DOI: 10.1103/PhysRevB.96.125413

\section{INTRODUCTION}

Over the past decade a vast amount of work has been devoted to exploring novel topological phases of condensed matter [1-4]. The rapid progress in this field began with the pioneering work on two-dimensional (2D) topological insulators (TIs) protected by time-reversal symmetry [5,6]. Their valence band structure is characterized by a nonzero $Z_{2}$ topological invariant $v=1[7,8]$. HgTe quantum wells and $\mathrm{Bi}$ bilayers are well-known examples of 2D TIs [9-12]. The concept of time-reversal invariant TIs was soon extended to three-dimensional (3D) systems whose band structure can be divided according to a set of $Z_{2}$ topological invariants $\left(v_{0} ; v_{1}, v_{2}, v_{3}\right)$ [13-19]. In $2011 \mathrm{Fu}$ proposed another phase of condensed matter called topological crystalline insulator (TCI) whose topological property is protected by crystal point-group symmetries [20]. SnTe in the rock-salt structure is the first material that was proved to be a 3D TCI with a nonzero mirror Chern number [21-24]. In addition, several 2D materials with a mirror reflection plane were theoretically predicted to be TCIs [25-28].

Both time-reversal invariant TIs and TCIs exhibit robust edge (surface) modes crossing the bulk band gap. The emergence of such surface bands is ensured by the bulk-edge correspondence $[29,30]$. Moreover, considering the fact that the surface-state wave function is a superposition of evanescent waves satisfying a particular boundary condition at the surface and further that the evanescent waves are solutions of the bulk Schrödinger equation satisfying the generalized Bloch condition with a complex wave number, it is not surprising that the emergence of surface modes is ruled by bulk topological invariants. The energy bands of a bulk crystal extended to complex wave numbers comprise the complex band structure. Recently, the complex band structure of TIs has been studied with the aim of exploring the properties of the surface modes in the bulk band gap $[31,32]$. Unfortunately, one cannot derive bulk topological invariants from the complex wave number vs energy plot of the complex bands, in the same way as one cannot find topological invariants just by plotting the energy dispersion of Bloch states with real wave number in the Brillouin zone (BZ).
In order to derive bulk topological invariants, one needs to compute the properties of electron wave functions. For a bulk crystal with a space-inversion center, the $Z_{2}$ topological invariant can easily be computed by considering the parity eigenvalues of the occupied valence bands at time-reversal invariant momentum (TRIM) points [33]. The evaluation of the $Z_{2}$ invariant for a noncentrosymmetric system is more difficult since one needs to integrate the Berry connection over half the 2D BZ where the the gauge of the wave functions must be chosen appropriately [34]. Alternatively, one can determine topological invariants by following the flow of the Wannier charge centers (WCCs) of the valence bands along a path connecting two symmetry points in the BZ [35-38]. Also, topological invariants can be deduced from the flow of the edge modes appearing in the entanglement spectrum when the system is spatially partitioned into two parts by a cut plane $[39,40]$.

In a recent work [41], two of the present authors showed that the $Z_{2}$ topological invariant of band insulators can be derived from the real eigenvalues of the embedding potential of Inglesfield $[42,43]$ in a projected bulk band gap. It is a bulk quantity that can be computed from the wave functions of the generalized Bloch states. However, its numerical computation is rather involved since one needs a real-space formalism that enables an accurate description of one-electron wave functions on a boundary surface between two atomic layers [44-46].

In the present paper we demonstrate that, alternatively, topological invariants of band insulators can be derived from the eigenvalues of the embedding potential formulated with localized basis functions [called local orbitals (LOs) hereafter]. The advantage of the present formulation is that the LO based embedding potential can readily be calculated, once one has a tight-binding Hamiltonian describing the valence and conduction bands of a bulk crystal. One can adopt either simple model Hamiltonians or first-principles ones expanded in terms of atomic-orbitals-like basis functions or the maximally localized Wannier functions [47]. The LO based embedding potential which is also called "contact (lead) self-energy" is widely used for calculating the electron transport through nanostructures sandwiched between two 
leads [48-50]. Several efficient methods have been developed to calculate this quantity for large systems [51-53].

Given a crystal orientation, the embedding potential is a function of the planar wave vector $\mathbf{k}$ and the one-electron energy $\varepsilon$. As will be shown, the embedding potential becomes Hermitian when $\varepsilon$ lies within a projected bulk band gap. Its real eigenvalues exhibit distinctively different behavior depending on the topological property of the valence bands, when they are plotted along a k-space path linking two symmetry points in the surface BZ. This behavior permits an unambiguous determination of the topological invariants. In addition to the $Z_{2}$ invariant of time-reversal invariant TIs discussed in our previous work [41], we demonstrate here that the present method can also be applied for determining the mirror Chern number of TCIs.

The plan of the present paper is as follows. Section II provides various theoretical aspects. We begin with explaining how to construct the LO based embedding potential from the generalized Bloch states and discuss the properties of its eigenvalues within a projected bulk band gap. We present the relationship between the embedding potential and the surface Green's function and discuss the effects of time-reversal symmetry and the evaluation of topological invariants. In Secs. III and IV results are presented for the 2D TI represented by the Bernevig-Hughes-Zhang (BHZ) model [9] and for a 3D TCI, SnTe, respectively. The summary and the comparison with other methods for determining topological invariants are provided in Sec. V. In the Appendix, we present a calculation of an interface between two BHZ-model systems in order to illustrate the advantage of using the LO embedding potential in studying the interface between two topological materials. Unless otherwise stated, we use Hartree atomic units with $m_{e}=e=\hbar=1$ throughout the paper.

\section{THEORY}

\section{A. Localized basis set}

We consider either a $2 \mathrm{D}$ or a $3 \mathrm{D}$ bulk crystal having periodic one-electron potential. Given an arbitrary crystal orientation, the system can be regarded as an infinite pileup of lattice planes stacked perpendicularly to the crystal orientation. We choose the $z$ axis as the surface normal, whereas $\mathbf{x}=(x, y)$ are $2 \mathrm{D}$ spatial coordinates within the plane. In the present paper, a set of lattice planes incorporating all atoms in a bulk unit cell is called "layer". We denote 2D lattice vectors within the plane by $\mathbf{R}_{\|}$, while the lattice vector connecting two unit cells belonging to two nearest-neighbor layers is denoted by $\mathbf{d}=\left(\mathbf{d}_{\|}, d_{z}\right)$, with $d_{z}(>0)$ giving the thickness of a single layer.

We calculate eigenstates of the one-electron Hamiltonian of the system for a given planar wave vector $\mathbf{k}$ and a given energy $\varepsilon$. In doing so, we consider not only Bloch states propagating in the $z$ direction but also evanescent waves that decay toward either $z=+\infty$ or $-\infty$. To expand solutions of the Schrödinger equation, we use basis functions specified by $\mathbf{k}$, layer index $l$, and orbital index $n$,

$$
\left\langle\mathbf{r} \sigma \mid \chi_{l n}^{\mathbf{k}}\right\rangle=\frac{1}{\sqrt{M}} \sum_{\mathbf{R}_{\|}} e^{i \mathbf{k} \cdot\left(\mathbf{x}_{l n}+\mathbf{R}_{\|}\right)} f_{n}\left(\mathbf{r}-\mathbf{r}_{l n}-\mathbf{R}_{\|}, \sigma\right),
$$

where $\mathbf{r}=(\mathbf{x}, z), \sigma$ is the spin index, $M$ is the number of lattice points in the plane, and $f_{n}(\mathbf{r}, \sigma)(n=1,2, \ldots, N)$ denotes spatially localized two-component spinor basis functions. In Eq. (1), $\mathbf{r}_{l n}=\left(\mathbf{x}_{l n}, z_{l n}\right)$ specifying the center of the $n$th basis function in layer $l$ is related to the corresponding one in layer $0, \mathbf{r}_{l=0 n}$, by $\mathbf{r}_{l n}=\mathbf{r}_{l=0, n}+l \mathbf{d}$.

We assume that the basis set $\left\{\chi_{l n}^{\mathbf{k}}\right\}$ is orthonormal, although the generalization to the case of a nonorthogonal basis set can easily be made by introducing overlap matrices between basis functions [43,53]. Let us expand $|\phi\rangle$, an arbitrary solution of the Schrödinger equation with $\mathbf{k}$ and $\varepsilon$, by using $\left\{\chi_{l n}^{\mathbf{k}}\right\}$ as

$$
|\phi\rangle=\sum_{l, n}\left|\chi_{l n}^{\mathbf{k}}\right\rangle\left\langle\chi_{l n}^{\mathbf{k}} \mid \phi\right\rangle .
$$

$\left\langle\chi_{\ln }^{\mathbf{k}} \mid \phi\right\rangle$ satisfies

$$
\hat{H}_{l, l+1}^{\mathbf{k}} \tilde{\phi}(l+1)+\hat{H}_{l, l}^{\mathbf{k}} \tilde{\phi}(l)+\hat{H}_{l, l-1}^{\mathbf{k}} \tilde{\phi}(l-1)=\varepsilon \tilde{\phi}(l),
$$

where $\hat{H}_{l, l^{\prime}}^{\mathbf{k}}$ is an $N \times N$ hopping matrix whose $\left(n, n^{\prime}\right)$ element is given by $\left\langle\chi_{l n}^{\mathbf{k}}|\hat{H}| \chi_{l^{\prime} n^{\prime}}^{\mathbf{k}}\right\rangle$ with $\hat{H}$ being the one-electron Hamiltonian, and $\tilde{\phi}(l)$ denotes a column vector of length $N$,

$$
\tilde{\phi}(l)=\left(\left\langle\chi_{l 1}^{\mathbf{k}} \mid \phi\right\rangle,\left\langle\chi_{l 2}^{\mathbf{k}} \mid \phi\right\rangle, \ldots,\left\langle\chi_{l N}^{\mathbf{k}} \mid \phi\right\rangle\right)^{t} .
$$

In Eq. (3) we have assumed that $\hat{H}_{l, l^{\prime}}^{\mathbf{k}}$ is nonvanishing only up to nearest-neighbor layer interactions. If this is not the case, for example, if $\hat{H}_{l, l+2}^{\mathbf{k}}$ is not negligible, one should double the layer thickness by treating two successive layers as "single layer". In the periodic bulk system, $\hat{H}_{l, l+1}^{\mathbf{k}}, \hat{H}_{l, l}^{\mathbf{k}}$, and $\hat{H}_{l, l-1}^{\mathbf{k}}$ do not depend on layer index $l$. Hereafter, we will use symbols, $\hat{\mathcal{H}}_{01}^{\mathbf{k}}, \hat{\mathcal{H}}_{00}^{\mathbf{k}}$, and $\hat{\mathcal{H}}_{10}^{\mathbf{k}}$ to denote the bulk Hamiltonian matrices.

\section{B. Complex band structure}

For given $\mathbf{k}$ and $\varepsilon$, we calculate wave functions satisfying the generalized Bloch condition,

$$
\tilde{\phi}(l+1)=\mu \tilde{\phi}(l),
$$

with

$$
\mu=\exp \left(i q_{z} d_{z}\right)
$$

where $q_{z}=k_{z}+i \kappa_{z}$ is a complex wave number. Bloch waves correspond to wave functions with $\kappa_{z}=0$, while those with positive (negative) $\kappa_{z}$ are evanescent waves decaying toward $l=+\infty(-\infty)$. Plotting complex wave number $q_{z}$ as functions of $\mathbf{k}$ and $\varepsilon$ is known as the complex band structure of a bulk crystal.

To obtain generalized Bloch states satisfying Eq. (4), we introduce a $2 N \times 2 N$ transfer matrix $\hat{T}$, connecting wave functions on successive layers by

$$
\left(\begin{array}{c}
\tilde{\phi}(l+1) \\
\tilde{\phi}(l)
\end{array}\right)=\hat{T}\left(\begin{array}{c}
\tilde{\phi}(l) \\
\tilde{\phi}(l-1)
\end{array}\right) .
$$

From Eq. (3), one sees that $\hat{T}$ is given by [14,51]

$$
\hat{T}=\left(\begin{array}{cc}
\left(\hat{\mathcal{H}}_{01}^{\mathbf{k}}\right)^{-1}\left[\varepsilon \hat{I}-\hat{\mathcal{H}}_{00}^{\mathbf{k}}\right] & -\left(\hat{\mathcal{H}}_{01}^{\mathbf{k}}\right)^{-1} \hat{\mathcal{H}}_{10}^{\mathbf{k}} \\
\hat{I} & \hat{O}
\end{array}\right),
$$

where $\hat{I}$ and $\hat{O}$ are $N$-dimensional identity and zero matrices, respectively. From Eqs. (4) and (6), we see that the generalized Bloch states satisfy the eigenvalue equation of the transfer 
matrix. We denote the $i$ th eigenvalue of $\hat{T}$ by $\mu_{i}$ and the corresponding eigenvector [only the upper half, i.e, the column vector of length $N$ corresponding to $\tilde{\phi}(l)]$ by $\tilde{u}_{i}$ with $i$ ranging from 1 to $2 N$. Generalized Bloch states can be divided into two groups depending on their asymptotic behavior. Those with index $i=1,2, \ldots, N$ are understood to fulfill the outgoing boundary condition at $l=+\infty$, i.e, they either decay or propagate toward $l=+\infty\left(\left|\mu_{i}\right| \leqslant 1\right)$, whereas those with $i=N+1, \ldots, 2 N$ are understood to satisfy the outgoing boundary condition at $l=-\infty$, i.e, they propagate or decay toward $l=-\infty\left(\left|\mu_{i}\right| \geqslant 1\right)$.

\section{Local-orbital based embedding potential}

The embedding theory of Inglesfield [42] is based on the real-space representation of one-electron wave functions. For a semi-infinite crystal with boundary surface $S$, its central quantity "embedding potential" relates the value and normal derivative on $S$ of an arbitrary electron wave function $\psi(\xi)$ with $\mathbf{k}$ and $\epsilon$, which either propagates or decays toward the interior of the semi-infinite crystal, by

$$
2 \int_{S} \Sigma\left(\mathbf{k}, \epsilon, \xi, \xi^{\prime}\right) \psi\left(\xi^{\prime}\right) d \xi^{\prime}=\partial_{n} \psi(\xi),
$$

with a composite index $\xi=(\mathbf{x}, \sigma)$. In the present paper we define the embedding potential for LOs such that it bears a close similarity to Eq. (8).

We divide the whole crystal into the semi-infinite one on the left-hand side $\left(l \leqslant l_{0}\right)$ and that on the right-hand side $(l \geqslant$ $\left.l_{0}+1\right)$ and define the embedding potential that describes the effects of the right-hand-side semi-infinite crystal $\left(l \geqslant l_{0}+1\right)$ on the boundary layer $l_{0}$. Let $|\psi\rangle$ be an arbitrary wave function with the parallel wave vector $\mathbf{k}$ and energy $\varepsilon$ that satisfies the outgoing boundary condition at $l=+\infty$. We denote the value of $|\psi\rangle$ on layer $l$ by

$$
\tilde{\psi}(l)=\left(\left\langle\chi_{l 1}^{\mathbf{k}} \mid \psi\right\rangle,\left\langle\chi_{l 2}^{\mathbf{k}} \mid \psi\right\rangle, \ldots,\left\langle\chi_{l N}^{\mathbf{k}} \mid \psi\right\rangle\right)^{t} .
$$

We define the embedding potential $\hat{\Sigma}(\mathbf{k}, \varepsilon)$ by an $N \times N$ matrix that relates $\tilde{\psi}\left(l_{0}\right)$ and $\tilde{\psi}\left(l_{0}+1\right)$ via

$$
\hat{\Sigma}(\mathbf{k}, \varepsilon) \tilde{\psi}\left(l_{0}\right)=\hat{\mathcal{H}}_{01}^{\mathbf{k}} \tilde{\psi}\left(l_{0}+1\right) .
$$

As is seen, the normal derivative of the wave function in Eq. (8) is replaced by $\hat{\mathcal{H}}_{01}^{\mathbf{k}} \tilde{\psi}\left(l_{0}+1\right)$ on the right-hand side of Eq. (9). Since $\hat{\Sigma}(\mathbf{k}, \varepsilon)$ has the same dimension as $\hat{\mathcal{H}}_{01}$, it is appropriate to call this quantity a nonlocal "potential". Because generalized Bloch states $\tilde{u}_{i}$ with $i=1,2, \ldots, N$ defined in the preceding section satisfy the outgoing boundary condition at $l=+\infty$, all of them should fulfill the equation

$$
\hat{\Sigma}(\mathbf{k}, \varepsilon) \tilde{u}_{i}=\hat{\mathcal{H}}_{01}^{\mathbf{k}} \mu_{i} \tilde{u}_{i} .
$$

Thus, by introducing an $N \times N$ matrix made out of $N$ eigenvectors, $\hat{V}=\left(\tilde{u}_{1}, \tilde{u}_{2}, \ldots, \tilde{u}_{N}\right)$, and a diagonal matrix $\hat{M}$ with elements $\hat{M}_{i j}=\delta_{i j} \mu_{i}$, we have

$$
\hat{\Sigma}(\mathbf{k}, \varepsilon) \hat{V}=\hat{\mathcal{H}}_{01}^{\mathbf{k}} \hat{V} \hat{M} .
$$

Hence, we obtain

$$
\hat{\Sigma}(\mathbf{k}, \varepsilon)=\hat{\mathcal{H}}_{01}^{\mathbf{k}} \hat{V} \hat{M} \hat{V}^{-1},
$$

indicating that the embedding potential $\hat{\Sigma}$ is a purely bulk quantity determined by the generalized Bloch states of a bulk crystal. Since $\hat{\Sigma}$ does not depend on the choice of the boundary layer $l_{0}$, we choose $l_{0}=0$ for simplicity in the following.

Here we consider the current carried by an arbitrary wave function with $\mathbf{k}$ and $\varepsilon,|\psi\rangle$, that satisfies the outgoing boundary condition at $l=+\infty$. The current from layer 0 through layer 1 can be evaluated from the values of the wave function as

$$
\begin{aligned}
J & =2 \operatorname{Im}\left[\tilde{\psi}^{\dagger}(1) \hat{\mathcal{H}}_{10}^{\mathbf{k}} \tilde{\psi}(0)\right] \\
& =2 \operatorname{Im}\left[\left\{\hat{\mathcal{H}}_{01}^{\mathbf{k}} \tilde{\psi}(1)\right\}^{\dagger} \tilde{\psi}(0)\right] \\
& =\frac{1}{i} \tilde{\psi}^{\dagger}(0)\left[\hat{\Sigma}^{\dagger}(\mathbf{k}, \varepsilon)-\hat{\Sigma}(\mathbf{k}, \varepsilon)\right] \tilde{\psi}(0),
\end{aligned}
$$

where we used $\hat{\mathcal{H}}_{10}^{\mathbf{k}}=\left(\hat{\mathcal{H}}_{01}^{\mathbf{k}}\right)^{\dagger}$. This equation bears a close similarity to the current expression in the embedding theory of Inglesfield [see, for example, Eq. (8) in Ref. [41]]. As discussed in Ref. [41], Eq. (12) has an important consequence: If $\varepsilon$ is located in a projected bulk band gap at a given $\mathbf{k}$, i.e, if all generalized Bloch states are evanescent waves, $J$ must vanish regardless of the value of $\tilde{\psi}(0)$. This signifies that within a projected bulk band gap, the embedding potential $\hat{\Sigma}$ is Hermitian, namely, $\hat{\Sigma}=\hat{\Sigma}^{\dagger}$. In this case, all eigenvalues of the embedding potential are real.

\section{Relationship to surface Green's function}

Let us divide the whole crystal into two semi-infinite pieces on the left- and right-hand sides by switching off the coupling term between layers 0 and $1, \hat{\mathcal{H}}_{01}^{\mathbf{k}}$. We will show that the LO based embedding potential defined by Eq. (9) is identical with so-called contact (lead) self-energy [48-53] defined in terms of the surface Green's function of the semi-infinite crystal occupying the right half-space $(l \geqslant 1)$ and decoupled from that on the left-hand side $(l \leqslant 0)$. The semi-infinite crystal on the right-hand side $(l \geqslant 1)$ will be called "bulk truncated surface" hereafter.

Let $|\psi\rangle$ be an arbitrary bulk solution of the Schrödinger equation with parallel wave $\mathbf{k}$ and $\varepsilon$ that satisfies the outgoing boundary condition at $l=+\infty(|\psi\rangle$ is defined for $-\infty<l<$ $+\infty$ ). If $l \geqslant 1$, Eq. (3) can be rewritten as

$$
\sum_{l^{\prime} \geqslant 1}\left[\varepsilon \delta_{l, l^{\prime}} \hat{I}-\hat{\mathcal{H}}_{l, l^{\prime}}^{\mathbf{k}}\right] \tilde{\psi}\left(l^{\prime}\right)=\delta_{l, 1} \hat{\mathcal{H}}_{10}^{\mathbf{k}} \tilde{\psi}(0) .
$$

We see that the matrix in the square bracket on the left-hand side of Eq. (13) is the inverse of the Green's function of the bulk truncated surface, $\hat{G}(\mathbf{k}, \varepsilon)$. Thus, by operating $\hat{G}(\mathbf{k}, \varepsilon)$ on both sides of Eq. (13) from the left, we obtain

$$
\tilde{\psi}(l)=\hat{G}_{l, 1}(\mathbf{k}, \varepsilon) \hat{\mathcal{H}}_{10}^{\mathbf{k}} \tilde{\psi}(0),
$$

where $\hat{G}_{l, l^{\prime}}$ denotes an $N \times N$ Green's function matrix whose $\left(n, n^{\prime}\right)$ element is given by $\left\langle\chi_{l n}^{\mathbf{k}}|\hat{G}| \chi_{l^{\prime} n^{\prime}}^{\mathbf{k}}\right\rangle$. This equation is essentially the same as Eq. (6.7) in the book of Inglesfield [43] except that he considered a more general nonorthogonal LO basis set. By choosing $l=1$, we have

$$
\tilde{\psi}(1)=\hat{G}_{11}(\mathbf{k}, \varepsilon) \hat{\mathcal{H}}_{10}^{\mathbf{k}} \tilde{\psi}(0) .
$$

By comparing Eqs. (9) and (15) and noting that both hold for an arbitrary solution $|\psi\rangle$ satisfying the outgoing boundary 
condition at $l=+\infty$, we have

$$
\hat{\Sigma}(\mathbf{k}, \varepsilon)=\hat{\mathcal{H}}_{01}^{\mathbf{k}} \hat{G}_{11}(\mathbf{k}, \varepsilon) \hat{\mathcal{H}}_{10}^{\mathbf{k}} .
$$

In previous theories of the electron transport through an interface between two metal leads, the whole system was divided into the interface region and the two unperturbed semi-infinite leads on both sides [48-50]. By projecting the Green's function equation for the whole system to that for a smaller Hilbert space spanned by the basis set of the interface region, one can easily show that the effects of the semi-infinite lead on the right-hand side $(l \geqslant 1)$ can be expressed by the self-energy term Eq. (16) acting on the boundary layer of the interface region, $l=0$. Several authors developed efficient methods to express the Green's function of the semi-infinite lead [51-54], and thus, the contact self-energy. The present formulation provides an alternative viewpoint concerning the physical meaning of the LO based embedding potential. In particular, by starting with Eq. (9) rather than with Eq. (16), the present definition reveals a close similarity of the LO based embedding potential to that of Inglesfield [42].

\section{E. Embedding-potential eigenvalues}

In the following, we consider the embedding potential of a band insulator for energies within a projected bulk band gap $\varepsilon_{v}(\mathbf{k})<\varepsilon<\varepsilon_{c}(\mathbf{k})$ where $\varepsilon_{v}(\mathbf{k})$ denotes the upper edge of the projected bulk valence bands, while $\varepsilon_{c}(\mathbf{k})$ denotes the lower edge of the projected bulk conduction bands. As was shown in Sec. II C, the embedding potential $\hat{\Sigma}(\mathbf{k}, \varepsilon)$ becomes an $N \times N$ Hermitian matrix within the projected bulk band gap. Alternatively, one may prove this by making use of the expression (16). The Green's function in Eq. (16) can be expressed using the spectral representation as

$$
\hat{G}(\mathbf{k}, \varepsilon)=\sum_{i} \frac{\left|\Psi_{\mathbf{k} i}\right\rangle\left\langle\Psi_{\mathbf{k} i}\right|}{\varepsilon+i \eta-\varepsilon_{i}},
$$

where $\left|\Psi_{\mathbf{k} i}\right\rangle$ denotes the $i$ th eigenstate with energy $\varepsilon_{i}$ of the bulk truncated surface and $\eta$ is a positive infinitesimal. As far as $\varepsilon$ is in the projected bulk band gap, the factor $1 /\left(\varepsilon+i \eta-\varepsilon_{i}\right)$ gives rise to no imaginary part, so that $\hat{G}$, and consequently, the $N \times N$ matrix $\hat{G}_{11}$ is Hermitian. This implies that $\hat{\Sigma}=$ $\hat{\mathcal{H}}_{01}^{\mathbf{k}} \hat{G}_{11} \hat{\mathcal{H}}_{10}^{\mathbf{k}}$ is also Hermitian. The only exception occurs when the bulk truncated surface exhibits a surface band within the projected bulk band gap. In this case, the embedding potential $\hat{\Sigma}(\mathbf{k}, \varepsilon)$ exhibits poles of first order on the energy dispersion curve of the surface state, $\varepsilon=\varepsilon_{s}(\mathbf{k})$.

Let us denote the $p$ th real eigenvalue of $\hat{\Sigma}(\mathbf{k}, \varepsilon)$ by $\lambda_{p}(p=$ $1,2, \ldots, N)$. With each $\lambda_{p}$, one can associate an evanescent wave with $\mathbf{k}$ and $\varepsilon$ decaying toward $l=+\infty,\left|\varphi_{p}\right\rangle$. Its value on boundary layer $0, \tilde{\varphi}_{p}(0)$, satisfies the eigenvalue equation,

$$
\hat{\Sigma}(\mathbf{k}, \varepsilon) \tilde{\varphi}_{p}(0)=\lambda_{p} \tilde{\varphi}_{p}(0),
$$

while those on the subsequent layers with $l \geqslant 1$ are related to $\tilde{\varphi}_{p}(0)$ by

$$
\tilde{\varphi}_{p}(l)=\hat{V} \hat{M}^{l} \hat{V}^{-1} \tilde{\varphi}_{p}(0) .
$$

By combining Eqs. (9) and (18), one has

$$
\lambda_{p} \tilde{\varphi}_{p}(0)=\hat{\mathcal{H}}_{01}^{\mathbf{k}} \tilde{\varphi}_{p}(1)
$$

which indicates that $\left|\varphi_{p}\right\rangle$ is not a generalized Bloch state satisfying Eq. (4). Instead, since $\hat{\Sigma}(\mathbf{k}, \varepsilon)$ is Hermitian, $\left|\varphi_{p}\right\rangle$ $(p=1,2, \ldots, N)$ are evanescent waves that are orthogonal to one another on boundary layer 0 , i.e., one can choose the eigenfunctions such that they satisfy

$$
\left\{\tilde{\varphi}_{p}(0)\right\}^{\dagger} \tilde{\varphi}_{q}(0)=\delta_{p q} .
$$

With this normalization, $\hat{\Sigma}(\mathbf{k}, \varepsilon)$ is expressed as

$$
\hat{\Sigma}(\mathbf{k}, \varepsilon)=\sum_{p=1}^{N} \lambda_{p} \tilde{\varphi}_{p}(0)\left\{\tilde{\varphi}_{p}(0)\right\}^{\dagger},
$$

with its determinant given by

$$
\operatorname{det}[\hat{\Sigma}(\mathbf{k}, \varepsilon)]=\prod_{p=1}^{N} \lambda_{p} .
$$

As stated above, $\hat{G}_{11}$ in Eq. (16), and thus, $\hat{\Sigma}(\mathbf{k}, \varepsilon)$ exhibits a pole if $(\mathbf{k}, \varepsilon)$ is on the surface-state energy dispersion curve of the bulk truncated surface, $\varepsilon=\varepsilon_{s}(\mathbf{k})$. Equation (22) implies that one of the eigenvalues exhibits a pole on the energy dispersion curve, i.e., $\lambda_{p} \sim C_{1}\left[\varepsilon-\varepsilon_{s}(\mathbf{k})\right]^{-1}$ as $\varepsilon \rightarrow \varepsilon_{s}(\mathbf{k})$. On the other hand, from Eq. (11) we have

$$
\begin{aligned}
\operatorname{det}[\hat{\Sigma}(\mathbf{k}, \varepsilon)] & =\operatorname{det}\left[\hat{\mathcal{H}}_{01}\right] \operatorname{det}[\hat{V}] \operatorname{det}[\hat{M}] \operatorname{det}\left[\hat{V}^{-1}\right] \\
& =\operatorname{det}\left[\hat{\mathcal{H}}_{01}\right] \prod_{i=1}^{N} \mu_{i} .
\end{aligned}
$$

Since $\left|\mu_{i}\right|<1$ within the projected bulk band gap, the righthand side of Eq. (24) does not diverge when $\varepsilon \rightarrow \varepsilon_{S}(\mathbf{k})$. This indicates that there must be another embedding-potential eigenvalue $\lambda_{q}$ that behaves as $\lambda_{q} \sim C_{2}\left[\varepsilon-\varepsilon_{s}(\mathbf{k})\right]$ when $\varepsilon \rightarrow \varepsilon_{s}(\mathbf{k})$.

Equation (11) also implies that the pole of the embedding potential occurs when $\operatorname{det}[\hat{V}]=0$, i.e., when the generalized Bloch states $\tilde{u}_{i}(i=1,2, \ldots, N)$ are not linearly independent. In this case, one can choose linear coefficients $c_{i} \quad(i=$ $1,2, \ldots, N)$ such that $\sum_{i=1}^{N} c_{i} \tilde{u}_{i}=0$. Then, one can construct an evanescent wave $|\psi\rangle$ whose value on layer $l(l \geqslant 0)$ is defined by

$$
\tilde{\psi}(l)=\sum_{i=1}^{N} c_{i} \mu_{i}^{l} \tilde{u}_{i} .
$$

$|\psi\rangle$ satisfies the Schrödinger equation of the bulk truncated surface with $l \geqslant 1$ since $\tilde{\psi}(0)=0$, so that it gives the surfacestate wave function of the bulk truncated surface.

The embedding-potential eigenvalue possesses a property that its partial derivative with respect to energy $\varepsilon$ is negative, which can be proved by using Eqs. (16), (17), (18), and (21) as

$$
\begin{aligned}
\frac{\partial \lambda_{p}}{\partial \varepsilon} & =\frac{\partial}{\partial \varepsilon}\left[\left\{\tilde{\varphi}_{p}(0)\right\}^{\dagger} \hat{\Sigma}(\mathbf{k}, \varepsilon) \tilde{\varphi}_{p}(0)\right] \\
& =\left\{\tilde{\varphi}_{p}(0)\right\}^{\dagger} \frac{\partial \hat{\Sigma}(\mathbf{k}, \varepsilon)}{\partial \varepsilon} \tilde{\varphi}_{p}(0) \\
& =-\sum_{i} \frac{\left|\left\{\tilde{\varphi}_{p}(0)\right\}^{\dagger} \hat{\mathcal{H}}_{01}^{\mathbf{k}} \tilde{\Psi}_{\mathbf{k} i}(1)\right|^{2}}{\left(\varepsilon+i \eta-\varepsilon_{i}\right)^{2}}<0,
\end{aligned}
$$


where $\tilde{\Psi}_{\mathbf{k} i}(1)$ denotes the value of $\left|\Psi_{\mathbf{k} i}\right\rangle$ on layer $l=1$. Equation (26) will be used to discuss the surface-state energy dispersion with $\mathbf{k}$ in the subsequent sections.

\section{F. Time-reversal symmetry}

We rewrite Eq. (20) using the original mathematical symbols,

$$
\lambda_{p}\left\langle\chi_{0 n}^{\mathbf{k}} \mid \varphi_{p}\right\rangle=\sum_{n^{\prime}=1}^{N}\left\langle\chi_{0 n}^{\mathbf{k}}|\hat{H}| \chi_{1 n^{\prime}}^{\mathbf{k}}\right\rangle\left\langle\chi_{1 n^{\prime}}^{\mathbf{k}} \mid \varphi_{p}\right\rangle .
$$

We take complex conjugate of the above equation. By noting that $\lambda_{p}$ is real and making use of $\langle\hat{T} f \mid \hat{T} g\rangle=\langle f \mid g\rangle^{*}$ with $\hat{T}$ denoting the time-reversal operator, we have

$$
\lambda_{p}\left\langle\hat{T} \chi_{0 n}^{\mathbf{k}} \mid \hat{T} \varphi_{p}\right\rangle=\sum_{n^{\prime}=1}^{N}\left\langle\hat{T} \chi_{0 n}^{\mathbf{k}}\left|\hat{T} \hat{H} \hat{T}^{-1}\right| \hat{T} \chi_{1 n^{\prime}}^{\mathbf{k}}\right\rangle\left\langle\hat{T} \chi_{1 n^{\prime}}^{\mathbf{k}} \mid \hat{T} \varphi_{p}\right\rangle .
$$

While the two orthonormal basis sets, $\left\{\hat{T} \chi_{0 n}^{\mathbf{k}}\right\}$ and $\left\{\chi_{0 n}^{-\mathbf{k}}\right\}$, are not necessarily identical, we assume that they are related to each other by a unitary transformation. Then, Eq. (28) reads

$$
\lambda_{p}\left\langle\chi_{0 n}^{-\mathbf{k}} \mid \hat{T} \varphi_{p}\right\rangle=\sum_{n^{\prime}=1}^{N}\left\langle\chi_{0 n}^{-\mathbf{k}}\left|\hat{T} \hat{H} \hat{T}^{-1}\right| \chi_{1 n^{\prime}}^{-\mathbf{k}}\right\rangle\left\langle\chi_{1 n^{\prime}}^{-\mathbf{k}} \mid \hat{T} \varphi_{p}\right\rangle .
$$

Now, we assume that the bulk crystal under consideration is time-reversal invariant, i.e., $\hat{T} \hat{H} \hat{T}^{-1}=\hat{H}$. In this case, $\left|\hat{T} \varphi_{p}\right\rangle$ becomes a solution of the bulk Schrödinger equation (evanescent wave) with energy $\varepsilon$ and parallel wave vector $-\mathbf{k}$ decaying toward $l=+\infty$. With the notation $\left|\hat{T} \varphi_{p}\right\rangle=\left|\varphi_{p}^{t r}\right\rangle$, Eq. (29) now reads

$$
\lambda_{p} \tilde{\varphi}_{p}^{t r}(0)=\hat{\mathcal{H}}_{01}^{-\mathbf{k}} \tilde{\varphi}_{p}^{t r}(1) .
$$

On the other hand, since $\left|\varphi_{p}^{t r}\right\rangle$ satisfies the outgoing boundary condition at $l=+\infty, \tilde{\varphi}_{p}^{t r}(0)$ and $\tilde{\varphi}_{p}^{t r}(1)$ are related by the embedding potential,

$$
\hat{\Sigma}(-\mathbf{k}, \varepsilon) \tilde{\varphi}_{p}^{t r}(0)=\hat{\mathcal{H}}_{01}^{-\mathbf{k}} \tilde{\varphi}_{p}^{t r}(1) .
$$

Combining the above two equations yields

$$
\hat{\Sigma}(-\mathbf{k}, \varepsilon) \tilde{\varphi}_{p}^{t r}(0)=\lambda_{p} \tilde{\varphi}_{p}^{t r}(0),
$$

which indicates that $\lambda_{p}$ is an eigenvalue of $\hat{\Sigma}(-\mathbf{k}, \varepsilon)$, namely,

$$
\lambda_{p}(-\mathbf{k}, \varepsilon)=\lambda_{p}(\mathbf{k}, \varepsilon) .
$$

The wave vector $\mathbf{k}$ is called a time-reversal invariant momentum (TRIM) point when $\mathbf{k}$ and $-\mathbf{k}$ are related by $\mathbf{k}=(-\mathbf{k})+\mathbf{G}_{\|}$with $\mathbf{G}_{\|}$being a reciprocal lattice vector contained in the lattice plane of a single layer. From Eq. (33), we see that the eigenstates of the embedding potential form doubly degenerate Kramers pairs consisting of two orthogonal states, $\left|\varphi_{p}\right\rangle$ and $\left|\varphi_{p}^{t r}\right\rangle$, at the TRIM points.

\section{G. Surface states}

Here, we would like to show that $\tilde{\varphi}_{p}(0)$ satisfying the eigenvalue equation Eq. (18) determines surface-state wave functions of a semi-infinite surface with a particular boundary condition. For this purpose, we introduce a semi-infinite system with layer index $l \geqslant 0$ in which the Hamiltonian matrices $\hat{H}_{l, l^{\prime}}^{\mathbf{k}}\left(l, l^{\prime} \geqslant 0\right)$ are assumed to be the same as those in the bulk except for the surface diagonal component $\hat{H}_{00}^{\mathbf{k}}$, which is assumed to be $\hat{H}_{00}^{\mathbf{k}}=\varepsilon_{a} \hat{I}$ with $\varepsilon_{a}$ being an arbitrary constant. Layer 0 may be interpreted as an overlayer with a constant on-site energy $\varepsilon_{a}$ adsorbed on a substrate represented by the bulk truncated surface with $l \geqslant 1$. For this Hamiltonian, we search a surface state $|\psi\rangle$ with wave vector $\mathbf{k}$ and a yet unknown energy $\varepsilon$ within the projected bulk band gap $\varepsilon_{v}(\mathbf{k})<\varepsilon<\varepsilon_{c}(\mathbf{k})$.

Since the Hamiltonian matrices are the same as those in the bulk except for $\hat{H}_{00}^{\mathbf{k}}, \tilde{\psi}(l)$ for $l \geqslant 1$ can be expressed by a linear combination of the $N$ evanescent waves $\left(\mu_{1}^{l} \tilde{u}_{1}, \mu_{2}^{l} \tilde{u}_{2}, \ldots, \mu_{N}^{l} \tilde{u}_{N}\right)$ with $\mathbf{k}$ and $\varepsilon$ discussed in Sec. II B. By choosing its unknown value on layer 0 as $\tilde{\psi}(0)$, their values on the subsequent layers are given by

$$
\tilde{\psi}(l)=\hat{V} \hat{M}^{l} \hat{V}^{-1} \tilde{\psi}(0) .
$$

For any values of $\tilde{\psi}(0)$, Eq. (34) satisfies the Schrödinger equation,

$$
\sum_{l^{\prime} \geqslant 0} \hat{H}_{l, l^{\prime}}^{\mathbf{k}} \tilde{\psi}\left(l^{\prime}\right)=\varepsilon \tilde{\psi}(l),
$$

except for $l=0$. Now, we would like to determine the unknown energy $\varepsilon$ and $\tilde{\psi}(0)$ such that Eq. (35) holds also for layer 0 , i.e.,

$$
\varepsilon_{a} \tilde{\psi}(0)+\hat{\mathcal{H}}_{01}^{\mathbf{k}} \tilde{\psi}(1)=\varepsilon \tilde{\psi}(0) .
$$

By substituting the relation

$$
\hat{\mathcal{H}}_{01}^{\mathbf{k}} \tilde{\psi}(1)=\hat{\Sigma}(\mathbf{k}, \varepsilon) \tilde{\psi}(0),
$$

in Eq. (36), we have

$$
\hat{\Sigma}(\mathbf{k}, \varepsilon) \tilde{\psi}(0)=\left(\varepsilon-\varepsilon_{a}\right) \tilde{\psi}(0) .
$$

By comparing Eqs. (18) and (38), we see that for a given $\mathbf{k}$, the surface state energy $\varepsilon$ is obtained by solving the equation,

$$
\lambda_{p}(\mathbf{k}, \varepsilon)=\varepsilon-\varepsilon_{a}(p=1,2, \ldots, N) .
$$

The corresponding surface state wave function on layer 0 is given by the $p$ th eigenvector of $\hat{\Sigma}(\mathbf{k}, \varepsilon)$, i.e., $\tilde{\psi}(0)=\tilde{\varphi}_{p}(0)$, and those on subsequent layers are calculated by Eq. (34).

In the following we consider a k-space path connecting two symmetry points in the SBZ. For simplicity, we use a scalar variable $k$ to represent $\mathbf{k}$ on the path. It is useful to consider a $3 \mathrm{D}$ orthogonal coordinate system $(k, \varepsilon, \lambda)$ with the $k \varepsilon$ plane chosen as the horizontal one and the $\lambda$ axis pointing in the vertical direction. Then, as schematically depicted in Fig. 1(a), one can plot eigenvalues of $\hat{\Sigma}(\mathbf{k}, \varepsilon)$ as 2D surfaces $\lambda=\lambda_{p}(k, \varepsilon)(p=1,2, \ldots, N)$ in the $3 \mathrm{D}(k, \varepsilon, \lambda)$ space. With this coordinate system, Eq. (39) becomes the equation for the line of intersection between two 2D surfaces, $\lambda=\lambda_{p}(k, \varepsilon)$ and $\lambda=\varepsilon-\varepsilon_{a}$. Figure 1(b) shows the cross-section view of these two surfaces on a vertical cut plane with a constant $k$, illustrating how the surface-state energy $\varepsilon_{s}(k)$ is graphically solved.

Using Eq. (39) we find that the gradient of the surface-state energy dispersion curve is given by

$$
\frac{d \varepsilon_{s}}{d k}=\frac{\partial \lambda_{p}\left(k, \varepsilon_{s}\right)}{\partial k}\left[1-\frac{\partial \lambda_{p}\left(k, \varepsilon_{s}\right)}{\partial \varepsilon}\right]^{-1} .
$$




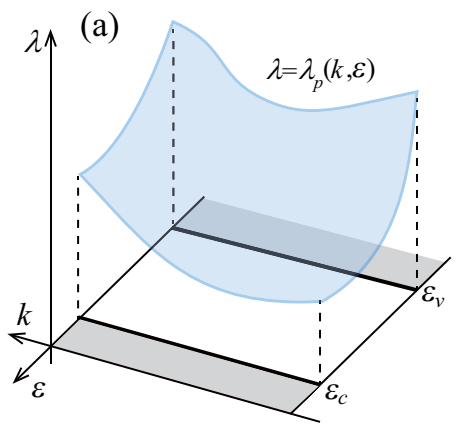

(b)

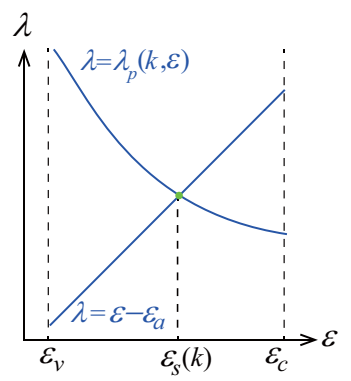

(c)

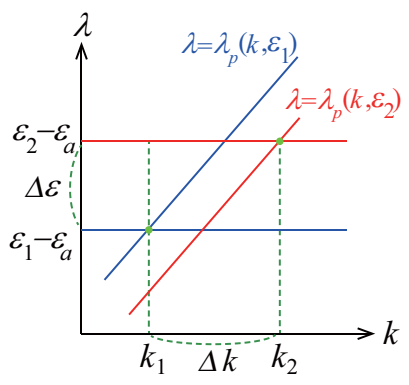

(d)

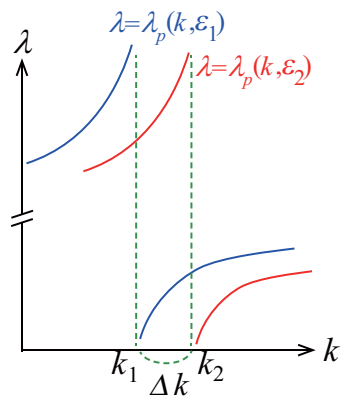

(a) $v=0$

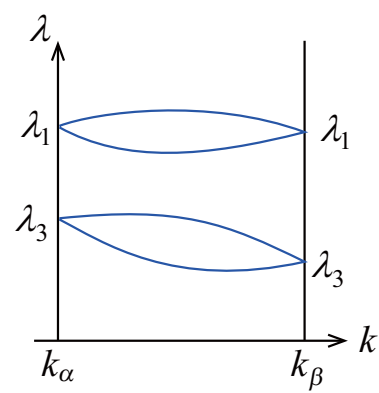

(b) $v=1$

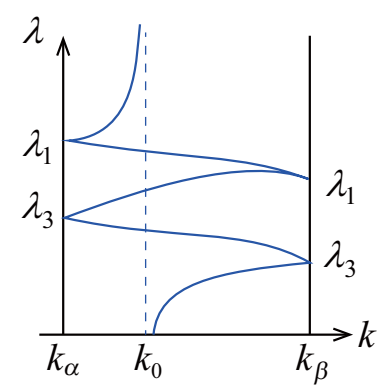

FIG. 1. (a) Schematic view of $2 \mathrm{D}$ surface $\lambda=\lambda_{p}(k, \epsilon)$ in 3D $(k, \epsilon, \lambda)$ coordinate space along a k-space path in the SBZ. This surface is defined only inside the projected bulk band gap $\epsilon_{v}(k)<\epsilon<\epsilon_{c}(k)$. (b) Cross-section view of two $2 \mathrm{D}$ surfaces, $\lambda=\lambda_{p}(k, \varepsilon)$ and $\lambda=$ $\varepsilon-\varepsilon_{a}$, on a plane with a constant $k$. The energy at the point of intersection between two lines gives $\varepsilon_{s}(k)$, the surface-state energy of a semi-infinite system in which an overlayer $(l=0)$ with constant site energy $\varepsilon_{a}$ is adsorbed on the bulk truncated surface $(l \geqslant 1)$ defined in the text. (c) Cross-section view of two $2 \mathrm{D}$ surfaces, $\lambda=\lambda_{p}(k, \varepsilon)$ and $\lambda=\varepsilon-\varepsilon_{a}$, on a plane with a constant energy $\left(\varepsilon_{1}\right.$ or $\left.\varepsilon_{2}\right)$. Two points, $\left(k_{1}, \varepsilon_{1}\right)$ and $\left(k_{2}, \varepsilon_{2}\right)$, are on the surface-state energy dispersion curve of the same surface as considered in panel (b). (d) Cross-section view of $\lambda=\lambda_{p}(k, \varepsilon)$ on a plane with a constant energy $\left(\varepsilon_{1}\right.$ or $\left.\varepsilon_{2}\right) \cdot \lambda=\lambda_{p}(k, \varepsilon)$ exhibits a pole at $k=k_{1}$ for $\varepsilon=\varepsilon_{1}$ and at $k=k_{2}$ for $\varepsilon=\varepsilon_{2}$. Two points, $\left(k_{1}, \varepsilon_{1}\right)$ and $\left(k_{2}, \varepsilon_{2}\right)$, are on the surface-state energy dispersion curve of the bulk truncated surface without an overlayer.

Since the denominator on the right-hand side of Eq. (40) is positive due to the inequality (26), $\frac{d \varepsilon_{s}}{d k}$ and $\frac{\partial \lambda_{p}\left(k, \varepsilon_{s}\right)}{\partial k}$ always have the same sign. This can be understood more easily by using a diagram. Let us consider the case with $\frac{\partial \lambda_{p}\left(k, \varepsilon_{s}\right)}{\partial k}>0$. Figure 1(c) shows the cross-section view of two surfaces, $\lambda=\lambda_{p}(k, \varepsilon)$ and $\lambda=\varepsilon-\varepsilon_{a}$, on a vertical cut plane with a constant energy $(\varepsilon=$ $\varepsilon_{1}$ or $\left.\varepsilon_{2}\right)$. Suppose that $\left(k_{1}, \varepsilon_{1}\right)$ is on $\varepsilon=\varepsilon_{s}(k)$. Then, two lines, $\lambda=\varepsilon_{1}-\varepsilon_{a}$ and $\lambda=\lambda_{p}\left(k, \varepsilon_{1}\right)$, intersect each other at $k=k_{1}$. Now, we increase the energy slightly from $\varepsilon_{1}$ to $\varepsilon_{2}=\varepsilon_{1}+\Delta \varepsilon$. Since $\frac{\partial \lambda_{p}(k, \varepsilon)}{\partial \varepsilon}<0$, the curve $\lambda=\lambda_{p}(k, \varepsilon)$ shifts downward with increasing $\varepsilon$, so that $\lambda=\lambda_{p}\left(k, \varepsilon_{2}\right)$ is located on the lower side of $\lambda=\lambda_{p}\left(k, \varepsilon_{1}\right)$. As a result, two lines, $\lambda=\varepsilon_{2}-\varepsilon_{a}$ and $\lambda=\lambda_{p}\left(k, \varepsilon_{2}\right)$, intersect each other at $k=k_{2}$, which is larger than $k_{1}$. This means that the gradient of the energy dispersion curve, $\Delta \varepsilon / \Delta k\left(\Delta k=k_{2}-k_{1}\right)$ is positive. In the same way one can show $\Delta \varepsilon / \Delta k<0$ if $\frac{\partial \lambda_{p}\left(k, \varepsilon_{s}\right)}{\partial k}<0$. Equation (40) indicates that the local behavior of the surface-state energy dispersion curve is ruled by the eigenvalue function $\lambda=\lambda_{p}(k, \varepsilon)$.

FIG. 2. (a) Lines of intersection between surfaces $\lambda=\lambda_{p}(k, \epsilon)$ $(p=1,2, \ldots, N)$ and a vertical cut plane $\epsilon=\epsilon_{0}$ for topologically trivial case with $v=0\left(\pi_{\alpha} \pi_{\beta}=1\right)$, where $\epsilon_{0}$ is located inside the projected band gap. Two states forming a Kramers pair at $k_{\alpha}$ meet again at $k_{\beta}$. (b) The same as (a) for a topologically nontrivial case with $v=1\left(\pi_{\alpha} \pi_{\beta}=-1\right)$. Two states forming a Kramers pair at $k_{\alpha}$ change partners at $k_{\beta}$.

We can make use of the inequality (26) again in discussing the surface-state energy dispersion curve on the bulk truncated surface, $\varepsilon=\varepsilon_{s}(k)$. Let us assume that $\varepsilon=\varepsilon_{s}(k)$ passes a point $\left(k_{1}, \varepsilon_{1}\right)$. In this case, as shown in Fig. $1(\mathrm{~d}), \lambda=\lambda_{p}\left(k, \varepsilon_{1}\right)$ as a function of $k$ exhibits a pole at $k=k_{1}$. In the vicinity of $k_{1}, \lambda_{p}\left(k, \varepsilon_{1}\right)$ is approximated by $\lambda \sim-A /\left(k-k_{1}\right)$ with a constant $A$ determining the sign of $\frac{\partial \lambda_{p}\left(k, \varepsilon_{1}\right)}{\partial k} \sim+A /\left(k-k_{1}\right)^{2}$ on both sides of the pole. In Fig. 1(d) it is assumed that $A>0$. Now, we increase the energy slightly from $\varepsilon_{1}$ to $\varepsilon_{2}=\varepsilon_{1}+\Delta \varepsilon$. Since $\frac{\partial \lambda_{p}(k, \varepsilon)}{\partial \varepsilon}<0$, the curve $\lambda=\lambda_{p}(k, \varepsilon)$ shifts downward with increasing $\varepsilon$. In this case, the pole of $\lambda=\lambda_{p}(k, \varepsilon)$ should shift gradually in the positive $k$ direction with increasing $\varepsilon$ and reach $k=k_{2}$, which is larger than $k_{1}$. As a result, $\Delta \varepsilon / \Delta k$ ( $\left.\Delta k=k_{2}-k_{1}\right)$, the gradient of $\varepsilon=\varepsilon_{s}(k)$ at $k=k_{1}$, becomes positive if $A>0$. In the same way one can show $\Delta \varepsilon / \Delta k<0$ if $A<0$.

\section{H. $Z_{2}$ topological invariants}

Let us consider a k-space path connecting two TRIM points in the SBZ, $\mathbf{k}_{\alpha}$ and $\mathbf{k}_{\beta}$. Figures 2(a) and 2(b) show schematically the lines of intersection obtained by cutting the 2D surfaces $\lambda=\lambda_{p}(k, \varepsilon)(p=1,2, \ldots, N)$ by a vertical cut plane $\varepsilon=\varepsilon_{0}$ with $\varepsilon_{0}$ being an arbitrary value in the projected bulk band gap. As mentioned in Sec. II F, these lines form $N / 2$ degenerate Kramers pairs at both ends, $k_{\alpha}$ and $k_{\beta}$. For the time being, we assume that the system obeys no other symmetry than time-reversal symmetry along the path. In this case, the $N$ lines $\lambda=\lambda_{p}\left(k, \varepsilon_{0}\right)(p=1,2, \ldots, N)$ will not intersect one another in the middle of the interval $\left[k_{\alpha}, k_{\beta}\right]$. Then, there should be two distinct ways these lines are connected between $k_{\alpha}$ and $k_{\beta}$ as shown in Figs. 2(a) and 2(b). In panel (a), two states in a Kramers pair at $k_{\alpha}$ meet again at $k_{\beta}$ to form a Kramers pair, whereas in panel (b), each state in a Kramers pair at $k_{\alpha}$ switches the partner at $k_{\beta}$. In the latter case, the set of line segments, $\lambda=\lambda_{p}\left(k, \varepsilon_{0}\right)(p=1,2, \ldots, N)$, forms a single continuous line alternating between $k_{\alpha}$ and $k_{\beta}$ and ranging between $\lambda=-\infty$ and $+\infty$, thus exhibiting necessarily a pole at $k=k_{0}$. 
In a recent work [41], two of the present authors revealed that one can determine the $Z_{2}$ topological invariant $v$ associated with two TRIM points $[13,33]$ by plotting the eigenvalues of the real-space embedding potential along a k-space path within a projected bulk band gap connecting the two points. Here, $v$ is related to the time-reversal polarizations at the two TRIM points, $\pi_{\alpha}$ and $\pi_{\beta}$, by $(-1)^{\nu}=\pi_{\alpha} \pi_{\beta}$. Similarly to Figs. 2(a) and 2(b), there are two distinct ways the embeddingpotential eigenvalues are connected between the two TRIM points, and it was revealed that panel (a) corresponds to $v=0$ and panel (b) to $v=1$. More generally, one has $v=P \bmod 2$ with $P$ the number of poles that the embedding potential exhibits along the $\mathbf{k}$-space path. Because of the close similarity between the LO based embedding potential and the real-space scheme of Inglesfield, we may safely say that the same criterion applies for the present LO based embedding potential.

So far, we have assumed that the system respects no other symmetry than time-reversal symmetry along the path connecting $k_{\alpha}$ and $k_{\beta}$. If this is not the case, for example, if the system is a TCI that respects certain crystal point-group operation along the $\mathbf{k}$-space path, then the $N$ lines $\lambda=\lambda_{p}\left(k, \varepsilon_{0}\right)(p=$ $1,2, \ldots, N)$ may be divided into different groups depending on their eigenvalues with respect to the point-group operation. Since the lines belonging to different eigenvalue groups can intersect one another without interaction, it might happen that the $N$ lines behave qualitatively differently from those in Figs. 2(a) and 2(b). Below we show that such a scenario indeed occurs for 3D TCIs.

\section{2D TI: BHZ MODEL}

We now demonstrate how the embedding-potential eigenvalues can be used for determining the topological invariants of band insulators. In the present section, we discuss the BHZ model representing a $2 \mathrm{D} Z_{2}$ TI [9]. In the following section, we discuss SnTe, a 3D TCI with a nonzero mirror Chern number $n_{M}[21]$.

The BHZ tight-binding Hamiltonian is a two-orbital model defined on a 2D square lattice [9]. To compute the embedding potential for its surface oriented in the [01] direction, the $x$ and $z$ axes are chosen to be parallel to the two sides of square unit cells. Then, as shown by a dashed line in Fig. 3(a), each "layer" becomes a one-dimensional (1D) lattice with a single lattice point per unit cell. Basis functions on each lattice point are specified by orbital index $\alpha=1,2$ and spin index $\sigma=$ $\uparrow(+1), \downarrow(-1)$, so that the number of basis functions per layer equals $N=4$. Furthermore, the BHZ Hamiltonian is diagonal with respect to spin index $\sigma$. Thus, the $4 \times 4$ matrix $\hat{\mathcal{H}}_{l, l^{\prime}}^{\mathbf{k}}$ defined in Sec. II A also becomes block diagonal with respect to $\sigma$. We denote the $2 \times 2$ submatrix for each spin component by $\hat{\mathcal{H}}_{l, l^{\prime}}^{k_{x}, \sigma}$, which contains the Hamiltonian matrix elements between two orbitals $\alpha=1,2$ with the same spin. They are given by

$$
\begin{aligned}
& \hat{\mathcal{H}}_{00}^{k_{x}, \sigma}=\left(\begin{array}{cc}
\varepsilon_{1}+2 t_{1} \cos k_{x} & 2 \sigma t_{12} \sin k_{x} \\
2 \sigma t_{12} \sin k_{x} & \varepsilon_{2}+2 t_{2} \cos k_{x}
\end{array}\right), \\
& \hat{\mathcal{H}}_{01}^{k_{x}, \sigma}=\left(\begin{array}{cc}
t_{1} & t_{12} \\
-t_{12} & t_{2}
\end{array}\right), \quad \hat{\mathcal{H}}_{10}^{k_{x}, \sigma}=\left(\begin{array}{cc}
t_{1} & -t_{12} \\
t_{12} & t_{2}
\end{array}\right) \text {, }
\end{aligned}
$$

(a) $\quad l-1 \quad l \quad l+1 \quad l+2$

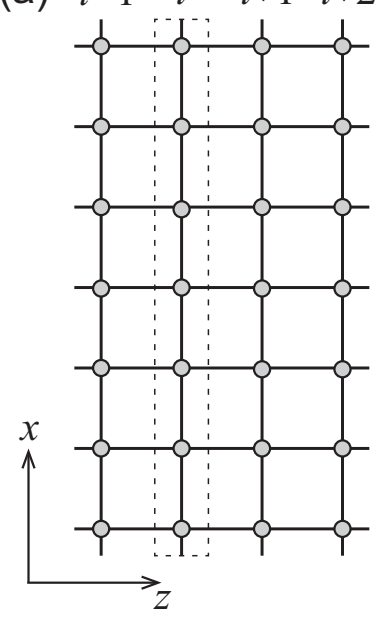

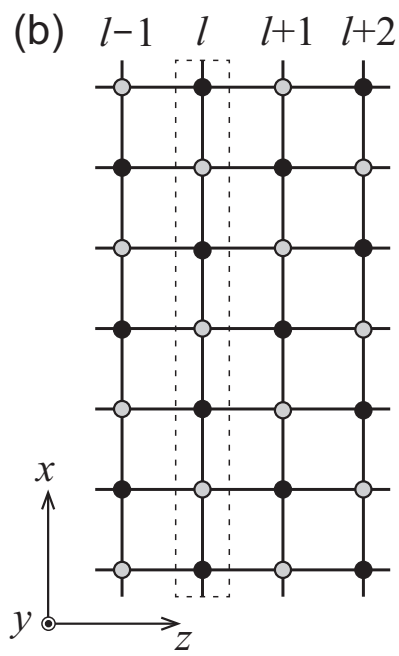

FIG. 3. (a) Bernevig-Hughes-Zhang model on a 2D square lattice. (b) $\mathrm{SnTe}$ or PbTe crystallized in rock-salt structure.

where $\varepsilon_{\alpha}\left(\varepsilon_{2}>\varepsilon_{1}\right)$ and $t_{\alpha}\left(t_{2}>0>t_{1}\right)$ are the site energies and the nearest-neighbor hopping integrals for orbitals $\alpha=$ 1,2 , while $t_{12}$ denotes the magnitude of the hopping integral between orbital 1 at the origin and orbital 2 on one of its four nearest-neighbor sites. Here we have chosen the lattice constant of the square lattice as unity. According to previous theoretical works [31,33], the present model represents a trivial band insulator $(v=0)$ if the $\alpha=1$ and 2 bands do not overlap energetically, namely, if the inequality

$$
\varepsilon_{2}-\varepsilon_{1}>4\left(\left|t_{1}\right|+\left|t_{2}\right|\right)
$$

holds. On the other hand, if the inequality sign in the above equation is reversed, the system becomes a 2D TI with $v=1$.

\section{A. Embedding potential eigenspectrum}

We consider two parameter sets to illustrate distinct phases. For the topological phase with $v=1$, we choose model parameters as $\varepsilon_{1}=-1.5, \varepsilon_{2}=1.5, t_{1}=-1, t_{2}=1$, and $t_{12}=0.5$. For the topologically trivial phase with $v=0$, we choose them as $\varepsilon_{1}=-1.5, \varepsilon_{2}=1.5, t_{1}=-0.15, t_{2}=0.15$, and $t_{12}=0.5$. In part I of the Supplemental Material [55] we present a detailed discussion of the complex band structure of both systems.

Solid and dashed lines in Fig. 4 show the embeddingpotential eigenvalues of the $\mathrm{BHZ}$ Hamiltonian along a constant energy path $\varepsilon=0.5$ within the projected bulk band gap over the whole BZ, $-\pi \leqslant k_{x} \leqslant \pi$. Panels (a) and (b) correspond to the aforementioned two cases with $v=1$ and 0 , respectively. Here, we have chosen to plot $\tan ^{-1} \lambda_{p}$ rather than $\lambda_{p}$ in order to be able to better show its behavior near the poles: In the vicinity of a pole at $k_{x}=k_{0}, \lambda_{p}$ may behave as $\lambda_{p} \sim-A /\left(k_{x}-k_{0}\right)$, indicating that $\tan ^{-1} \lambda_{p} \rightarrow \pm \pi / 2$ while $\frac{\partial}{\partial k_{x}} \tan ^{-1} \lambda_{p} \rightarrow+1 / A$ as $k_{x} \rightarrow k_{0}$, so that the value of $\tan ^{-1} \lambda_{p}$ jumps by $\pi$ while its derivative with respect to $k_{x}$ remains the same, when $k_{x}$ crosses the pole.

Since the BHZ Hamiltonian is diagonal with respect to the spin index $\sigma$, the embedding potential $\hat{\Sigma}\left(k_{x}, \varepsilon\right)$ is also diagonal with respect to $\sigma$, and $\hat{\Sigma}\left(k_{x}, \varepsilon\right)$ for each spin component has 

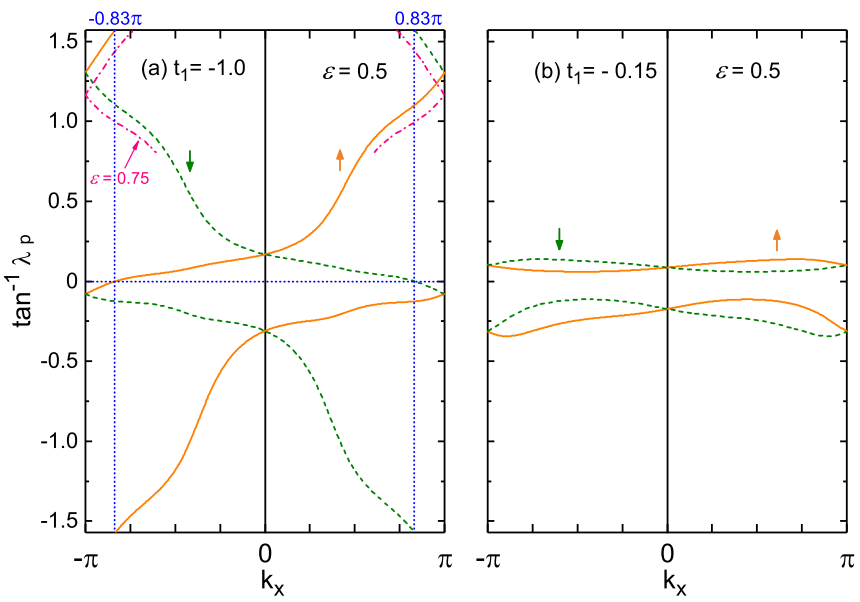

FIG. 4. Embedding-potential eigenvalues of the BHZ Hamiltonian, $\lambda_{p}\left(k_{x}, \varepsilon\right)(p=1$ to 4$)$, as a function of $k_{x}$ at $\varepsilon=0.5$. In panel (a), $\varepsilon_{1}=-1.5, \varepsilon_{2}=1.5, t_{1}=-1, t_{2}=1$, and $t_{12}=0.5$, indicating $Z_{2}$ topological invariant $v=1$. The parameters in (b) are the same as those in (a) except for $t_{1}=-0.15$ and $t_{2}=0.15$, implying $v=0$. Orange (solid) and green (dashed) lines represent eigenvalues for up-spin and down-spin electrons, respectively. The red (dot-dashed) lines in panel (a) show the two uppermost branches of $\lambda_{p}\left(k_{x}, \varepsilon\right)$ at a higher energy $\varepsilon=0.75$ near $k_{x}= \pm \pi$.

two eigenvalues at a given $k_{x}$. In contrast to the complex band structure [55], these eigenvalues are not degenerate with respect to spin index $\sigma$. The eigenstates of the up-spin component at $k_{x}$ and those of the down-spin component at $-k_{x}$ are related by time-reversal operation. Hence, at the two TRIM points, $k_{x}=0$ and $\pi(-\pi)$, two eigenstates with both spin components form a degenerate Kramers pair. In panel (a), each eigenstate in a Kramers pair at $k_{x}=0$ switches the partner at $k_{x}=\pi$, thus indicating $v=1$, whereas in panel (b) two states in a Kramer pair at $k_{x}=0$ meet again at $k_{x}=\pi$, thus indicating $v=0$. These results are in full accord with the discussion in Sec. II H concerning the behavior of the embedding-potential eigenvalues along a path linking two TRIM points.

To illustrate the energy dependence of the embeddingpotential eigenvalues, we also plotted two uppermost branches of the $\lambda_{p}$ values at a slightly larger energy $\varepsilon=0.75$ (only near $k_{x}= \pm \pi$ ) by dot-dashed lines in Fig. 4(a). As discussed in Sec. II G, $\lambda_{p}$ shifts downward monotonously with increasing $\varepsilon$. As a consequence, the pole of the highest branch at $k_{x}=-0.83 \pi(+0.83 \pi)$ shifts to a larger (smaller) $k_{x}$ value with increasing $\varepsilon$, which agrees with the schematic diagram shown in Fig. 1(d).

Here, it should be remarked that as discussed in Sec. IIE, when one of the embedding-potential eigenvalues exhibits a pole at $\left(k_{0}, \varepsilon_{0}\right)$, there must be another eigenvalue that tends to zero as $\left(k_{x}, \varepsilon\right) \rightarrow\left(k_{0}, \varepsilon_{0}\right)$. Indeed, as seen from Fig. 4(a), one eigenvalue of the up-spin (down-spin) component tends to zero as $k_{x} \rightarrow-0.83 \pi(+0.83 \pi)$.

In Fig. 4, we have chosen on-site energies of two orbitals as $\varepsilon_{1}=-1.5$ and $\varepsilon_{2}=1.5$. In this case, the $Z_{2}$ topological invariant should undergo a transition between $v=1$ and $v=0$ when $\left|t_{1}\right|+\left|t_{2}\right|$ goes across the critical value $\left(\varepsilon_{2}-\varepsilon_{1}\right) / 4=0.75$ as indicated by Eq. (43). In part II of the Supplemental Material
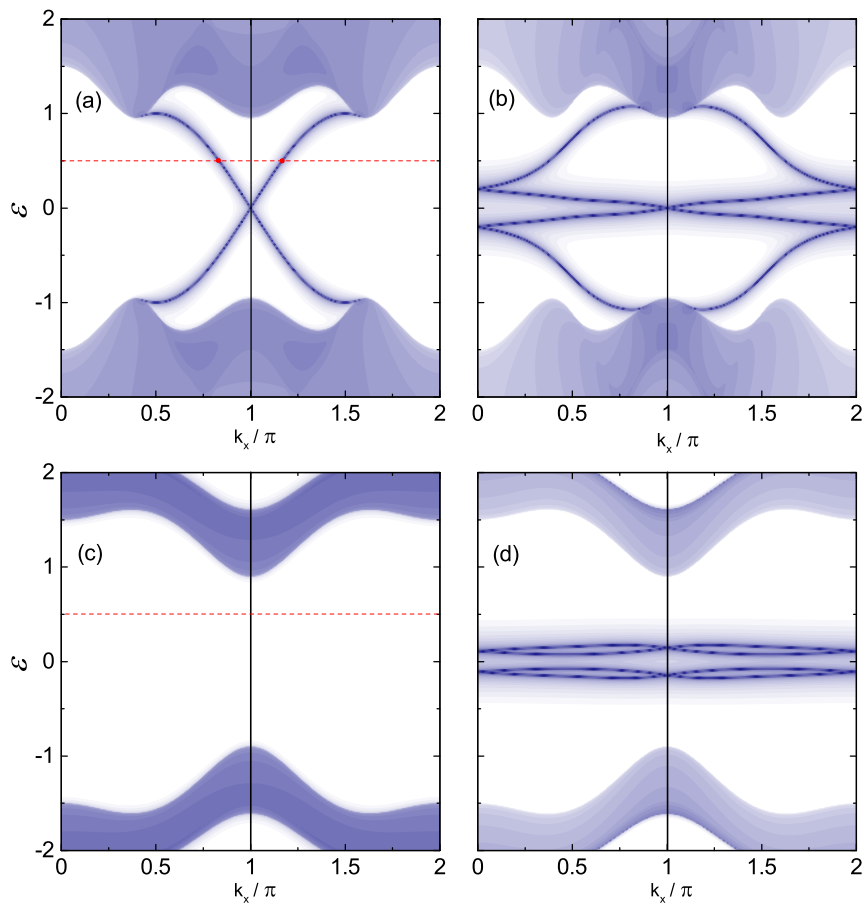

FIG. 5. (a) Intensity plot of $\rho_{1}\left(k_{x}, \varepsilon\right), k_{x}$-resolved DOS of the outermost layer, for the bulk truncated surface with $\varepsilon_{1}=-1.5, \varepsilon_{2}=$ $1.5, t_{1}=-1, t_{2}=1$, and $t_{12}=0.5$. (b) $\rho_{0}\left(k_{x}, \varepsilon\right), k_{x}$-resolved DOS of the overlayer $(l=0)$ with on-site energy $\varepsilon_{a}=0.0$ adsorbed on top of the bulk truncated surface in panel (a). (c) The same as (a) except for $t_{1}=-0.15$ and $t_{2}=0.15$. (d) The same as (b) adsorbed on top of the bulk truncated surface in panel (c). In all panels, dark and bright regions represent projected bulk bands and projected bulk band gaps, respectively. Discrete bands appearing in projected band gaps are localized surface states. Small imaginary energy $\gamma=10^{-3}$ is used to broaden the $\delta$-function-like peaks of surface bands. Dashed (red) lines in panels (a) and (c) indicate constant energy path $\varepsilon=0.5$, along which the embedding-potential eigenvalues in Fig. 4 are plotted.

[55] we discuss how the embedding-potential eigenvalues behave in the vicinity of this critical value.

\section{B. Local density of states}

In Fig. 4(a), the embedding-potential eigenvalues exhibit two poles at $k_{x}= \pm 0.83 \pi$. As discussed in Sec. IIE, this indicates that localized surface states emerge on the bulk truncated surface and their energy dispersion curve $\varepsilon=\varepsilon_{s}\left(k_{x}\right)$ intersects $\varepsilon=0.5$ at $k_{x}= \pm 0.83 \pi$. Furthermore, the gradient of the dispersion curve at $k_{x}=-0.83 \pi(+0.83 \pi)$ should be positive (negative). To confirm this, we calculated the $k_{x}$-resolved density of states (DOS) of layer $l$ defined by

$$
\rho_{l}(\mathbf{k}, \varepsilon)=-\frac{1}{\pi} \operatorname{Im} \operatorname{Tr} \hat{G}_{l, l}(\mathbf{k}, \varepsilon+i \gamma) .
$$

In Fig. 5(a) we show the intensity plot of the DOS of the outermost layer, $\rho_{1}\left(k_{x}, \varepsilon\right)$, with the same parameter set as used in Fig. 4(a), where a small imaginary energy $\gamma=$ $10^{-3}$ was introduced to broaden $\delta$-function-like DOS peaks of localized surface bands. Since the present system is a topological insulator with $v=1$, gapless edge states emerge within the projected bulk band gap, and it is seen that their 
energy dispersion curves intersect $\varepsilon=0.5$ (dashed red line) at $k_{x}=0.83 \pi$ and $1.17 \pi(-0.83 \pi)$ and also that their slopes at $k_{x}=0.83 \pi$ and $1.17 \pi$ are negative and positive, respectively, as expected.

In Fig. 5(a) one has a single surface band for each spin component crossing the bulk band gap. Since there exist four embedding-potential eigenvalues at a given $k_{x}$, one can have more surface bands by modifying the boundary condition at the surface. As an example, we consider a semi-infinite surface introduced in Sec. II G in which a single layer $(l=0)$ expressed by a Hamiltonian matrix $\hat{H}_{00}^{\mathbf{k}}=\varepsilon_{a} \hat{I}$ is adsorbed on top of the same bulk truncated surface with $l \geqslant 1$. Figure 5(b) shows the calculated overlayer DOS $\rho_{0}\left(k_{x}, \varepsilon\right)$ for the case of $\varepsilon_{a}=0.0$. As shown in Fig. 1(b), the surface state energies at a given $k_{x}$ are those at the points of intersection between $\lambda=\lambda_{p}\left(k_{x}, \varepsilon\right)(p=1$ to 4$)$ and $\lambda=\varepsilon-\varepsilon_{a}=\varepsilon$. In the present case, each of the four eigenvalue curves intersects once the line $\lambda=\varepsilon$ in the energy gap, so that four surface bands emerge in the projected bulk band gap except for the vicinity of $k_{x}=\pi$.

Figure 5(c) shows the DOS of the outermost layer, $\rho_{1}\left(k_{x}, \varepsilon\right)$, for the bulk truncated surface with the same parameter as in Fig. 4(b). As is seen, this surface exhibits no surface states within the projected bulk band gap. Yet, it is possible to induce surface bands by modifying the boundary condition of wave functions at the surface. As an example we put an overlayer with $\varepsilon_{a}=0.0$ similarly to the case of Fig. 5(b). The overlayer DOS of the resultant surface, $\rho_{0}\left(k_{x}, \varepsilon\right)$, plotted in Fig. $5(\mathrm{~d})$ exhibits four surface bands throughout the SBZ $-\pi \leqslant k_{x} \leqslant \pi$. It is seen that two surface states in a Kramers pair at $k_{x}=0$ meet again at $k_{x}=\pi$, which is in accord with the $Z_{2}$ invariant $v=0$ for the present system.

\section{3D TCI}

As the second application, we consider tin telluride ( $\mathrm{SnTe}$ ) crystallized in a rock-salt structure. SnTe is a TCI whose topological state of matter is protected by the mirror symmetry about the (110) or equivalent lattice planes [21-24]. The results for this 3D TCI are also compared to those for the closely related trivial band insulator $\mathrm{PbTe}$.

Let us consider a 2D plane $K$ in the momentum space which is invariant with respect to mirror reflection operation $\hat{R}_{M}$, i.e., every wave vector on $K$ is mapped by $\hat{R}_{M}$ onto itself or onto an equivalent one differing only by a $3 \mathrm{D}$ reciprocal lattice vector. Bloch states whose 3D wave vector lies on $K$ can be labeled by a mirror parity eigenvalue, $+i$ or $-i$ (note that $\hat{R}_{M}^{2}=-\hat{I}$ because of $2 \pi$ rotation in the spin space). Hence, within the 2D BZ on $K$, the occupied valence bands are divided into two groups depending on whether their mirror parity eigenvalues are $+i$ or $-i$. For each of the two groups, one can define a topological invariant, $n_{+i}$ or $n_{-i}$, by summing up the Chern numbers of all the bands in the same group. Then, the mirror Chern number is defined by

$$
n_{M}=\left(n_{+i}-n_{-i}\right) / 2 .
$$

For time-reversal invariant systems, $n_{M}$ becomes an integer since $n_{+i}=-n_{-i}$ holds. If $n_{M}$ is nonzero, any crystal surface which is symmetric about the mirror operation $\hat{R}_{M}$ exhibits topologically protected gapless surface bands along the symmetry line obtained by projecting $K$ onto the SBZ.
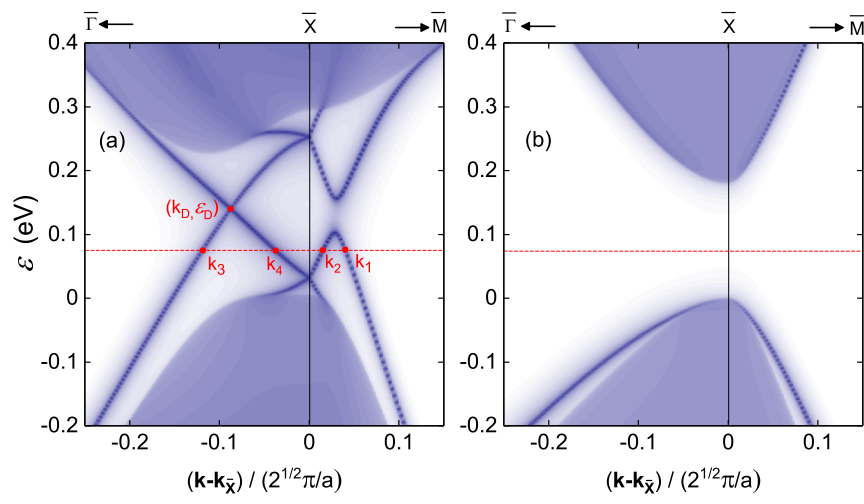

FIG. 6. (a) Intensity plot of the k-resolved DOS for the outermost layer, $\rho_{1}(\mathbf{k}, \varepsilon)$, of a bulk truncated $\operatorname{SnTe}(001)$ surface along the $\bar{\Gamma}-\bar{X}$ and $\bar{X}-\bar{M}$ lines. (b) The same as (a) for $\mathrm{PbTe}(001)$. Small imaginary energy $\gamma=10^{-3} \mathrm{eV}$ is used to broaden the $\delta$-function-like peaks of surface bands.

It was shown that $\mathrm{SnTe}$ exhibits $n_{M}=-2$ with regard to the (110) and equivalent mirror planes [21].

We calculate the embedding-potential eigenvalues of SnTe for the (001) surface. As shown in Fig. 3(b), each "layer" contains a single (001) lattice plane forming a square lattice with a unit cell containing a $\mathrm{Sn}$ atom at $\mathbf{x}=(0,0)$ and a $\mathrm{Te}$ atom at $\mathbf{x}=\left(\frac{a}{2}, 0\right)$ with $a$ being the cubic lattice constant of SnTe. With this choice of the coordinate system, the four TRIM points in the SBZ are $\mathbf{k}_{\bar{\Gamma}}=(0,0), \mathbf{k}_{\bar{X}}=\left(\frac{\pi}{a}, \frac{\pi}{a}\right), \mathbf{k}_{\bar{Y}}=\left(-\frac{\pi}{a}, \frac{\pi}{a}\right)$ (equivalent to $\bar{X})$, and $\mathbf{k}_{\bar{M}}=\left(0, \frac{2 \pi}{a}\right)$. SnTe is topologically trivial with respect to time-reversal symmetry, having the $3 \mathrm{D}$ $Z_{2}$ invariants $\left(v_{0} ; v_{1} v_{2} v_{3}\right)=(0 ; 0,0,0)$ [33]. Thus, the $v$ values associated with the $\bar{\Gamma}-\bar{X}, \bar{\Gamma}-\bar{M}$, and $\bar{X}-\bar{M}$ lines are all $v=0$. The above three symmetry lines are invariant with respect to the mirror operation about the (110), (100), and (110) lattice planes, respectively, so that one can define the mirror Chern number $n_{M}$ for each of the three lines. Among them, only $n_{M}$ associated with the $\bar{\Gamma}-\bar{X}$ line has a nonzero value $n_{M}=-2$.

\section{A. Surface energy bands}

To describe the band structure of SnTe, we adopt the nearest-neighbor tight-binding model Hamiltonian proposed by Lent et al. [56] which incorporates the SO coupling. As basis functions they employed nine orbitals $\left(s, p_{x}, p_{y}, p_{z}\right.$, $d_{x^{2}-y^{2}}, d_{3 z^{2}-r^{2}}, d_{x y}, d_{y z}$, and $d_{z x}$ ) for each atom per spin. Thus, the number of basis functions per layer amounts to $N=$ $9 \times 2 \times 2=36$, indicating that the dimension of the embedding potential $\hat{\Sigma}(\mathbf{k}, \varepsilon)$ and the number of its real eigenvalues within the projected bulk band gap both are $N=36$. For comparison, we also consider isocrystalline telluride $\mathrm{PbTe}$ with vanishing mirror Chern number $n_{M}=0$. The tightbinding parameters of both materials are listed in Ref. [56].

Figure 6(a) shows the calculated DOS of the outermost layer, $\rho_{1}(\mathbf{k}, \varepsilon)$ defined by Eq. (44), for a semi-infinite bulk truncated $\mathrm{SnTe}(001)$ surface along the $\bar{\Gamma}-\bar{X}$ and $\bar{X}-\bar{M}$ lines, where we focus a small area near the $\bar{X}$ point where the projected bulk band gap becomes the smallest. In contrast to the corresponding DOS for PbTe(001) shown in panel (b), $\mathrm{SnTe}(001)$ exhibits two surface bands crossing the band gap 

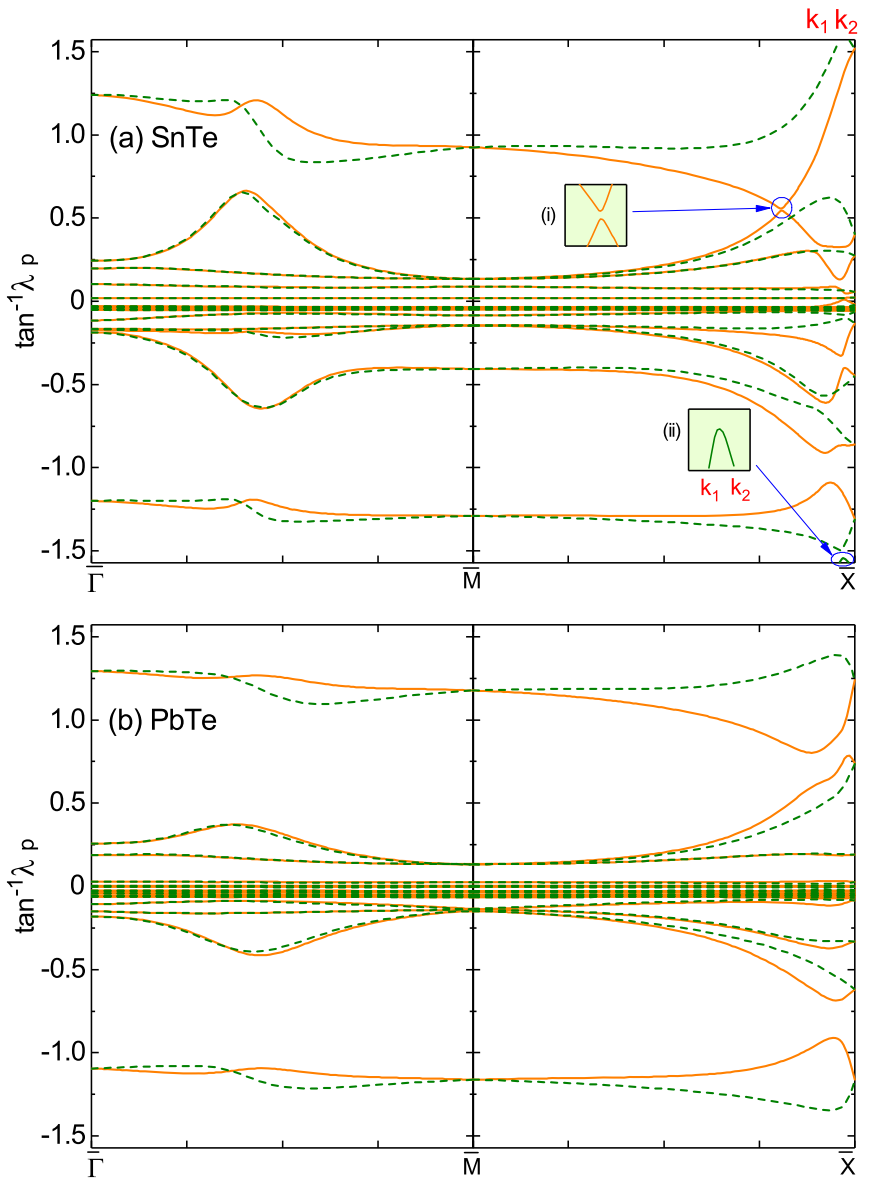

FIG. 7. Embedding-potential eigenvalues $\quad \lambda_{p}(\mathbf{k}, \varepsilon)$ ( $p=1,2, \ldots, N=36$ ) for (a) $\operatorname{SnTe}(001)$ and (b) $\operatorname{PbTe}(001)$ along the $\bar{\Gamma}-\bar{M}$ line (left half) and the $\bar{M}-\bar{X}$ line (right half) at an energy within the band gap, $\varepsilon=0.075 \mathrm{eV}$, indicated by red lines in Fig. 6. Two insets in panel (a) illustrate the enlargement of a small area indicated by blue arrows.

along the $\bar{\Gamma}-\bar{X}$ line. The two bands intersect each other without interactions at $\left(\mathbf{k}_{D}, \varepsilon_{D}\right)=\left(0.91 \mathbf{k}_{\bar{X}}, 0.14\right)$ forming a $2 \mathrm{D}$ Dirac point in the energy dispersion relation of the surface bands. Electron energy bands are periodic along the $\mathbf{k}$-space line passing $\bar{\Gamma}$ and $\bar{X}$, and one period (whole 1D BZ) is given by the interval, $-\mathbf{k}_{\bar{X}} \rightarrow \mathbf{k}_{\bar{\Gamma}} \rightarrow \mathbf{k}_{\bar{X}}$ with two end points related by a 2 D reciprocal lattice $\mathbf{G}_{\|}=\left(\frac{2 \pi}{a}, \frac{2 \pi}{a}\right)$. Because of time-reversal symmetry, another Dirac point emerges at $\left(-\mathbf{k}_{D}, \varepsilon_{D}\right)$.

\section{B. Embedding potential eigenspectrum}

We begin with the topologically trivial $\bar{\Gamma}-\bar{M}$ and $\bar{M}-\bar{X}$ lines. In Fig. 7 we plot the calculated $\lambda_{p}(\mathbf{k}, \varepsilon)$ values $(p=$ $1,2, \ldots, N=36$ ) for (a) $\operatorname{SnTe}(001)$ and (b) $\mathrm{PbTe}(001)$ along the $\bar{\Gamma}-\bar{M}-\bar{X}$ line on a constant energy path within the band gap, $\varepsilon=0.075 \mathrm{eV}$, which is indicated by red lines in Fig. 6 . As aforementioned, the $\mathbf{k}$ points along the $\bar{\Gamma}-\bar{M}$ and $\bar{M}-\bar{X}$ lines are invariant with respect to mirror operations about the (100) and (110) planes, respectively, so that $\lambda_{p}(\mathbf{k}, \varepsilon)$ can be divided into two groups according to whether the corresponding eigenfunction $\left|\varphi_{p}\right\rangle$ has mirror parity $+i$ or $-i$.
In Fig. 7, those with $+i$ are shown by solid (orange) lines, while those with $-i$ are shown by dashed (green) lines.

As discussed in Sec. II F, $\left|\varphi_{p}\right\rangle$ at $\mathbf{k}$ and its time-reversal state $\left|\hat{T} \varphi_{p}\right\rangle$ at $-\mathbf{k}$ form a Kramers pair having the same embedding-potential eigenvalue $\lambda_{p}$. It is easy to show that these two states have opposite mirror parity values. Namely, two states with $(\mathbf{k}, \pm i)$ and $(-\mathbf{k}, \mp i)$ emerge always as a pair in the $\mathbf{k}$ vs $\lambda_{p}$ plot along a symmetry line in the SBZ that is invariant with respect to mirror operation. This explains why doubly degenerate Kramers pairs at the three TRIM points, $\bar{\Gamma}, \bar{M}$, and $\bar{X}$, shown in Fig. 7 are always made out of two states with opposite mirror parity values. The $Z_{2}$ invariants associated with $\bar{\Gamma}-\bar{M}$ and $\bar{M}-\bar{X}$ are $v=0$ for both SnTe and $\mathrm{PbTe}$. As a result, two states in a degenerate Kramers pair at $\bar{\Gamma}$ meet again to form a Kramers pair at $\bar{M}$ along the $\bar{\Gamma}-\bar{M}$ line for both materials. This is in line with $v=0$. The same applies also for the $\bar{M}-\bar{X}$ line.

As seen in Fig. 6(a), the bulk truncated SnTe(001) surface exhibits surface bands along the $\bar{M}-\bar{X}$ line and one of them intersects $\varepsilon=0.075 \mathrm{eV}$ (red line) at $\mathbf{k}_{1}$ and $\mathbf{k}_{2}$. As discussed in $\mathrm{Sec}$. II E, the embedding-potential eigenvalues exhibit poles on the surface-state energy dispersion curves of a bulk truncated surface. Indeed, the uppermost eigenvalue curve with mirror parity $-i$ in Fig. 7(a) exhibits two poles at $\mathbf{k}_{1}$ and $\mathbf{k}_{2}$.

We now discuss the variation of $\lambda_{p}(\mathbf{k}, \varepsilon)$ along the $\bar{\Gamma}-\bar{X}$ direction. In Figs. 8(a) and 8(b), we show the calculated embedding-potential eigenvalues $\lambda_{p}(\mathbf{k}, \varepsilon)$ along the $\bar{\Gamma}-\bar{X}$ line at a constant energy $\varepsilon=0.075 \mathrm{eV}$ for (a) $\operatorname{SnTe}(001)$ and (b) $\mathrm{PbTe}(001)$, respectively. The $Z_{2}$ invariant associated with $\bar{\Gamma}-\bar{X}$ is $v=0$ for both materials. Thus, as expected, two states of a degenerate Kramers pair at $\bar{\Gamma}$ meet again to form a Kramers pair at $\bar{X}$ for $\mathrm{PbTe}(001)$. On the other hand, it is surprising to see that two states in a degenerate Kramers pair at $\bar{\Gamma}$ switch partners at the $\bar{X}$ point for $\operatorname{SnTe}(001)$ in panel (a). Looking into more details, one sees that two states forming a Kramers pair at $\bar{\Gamma}$ switch partners and belong to two Kramers pairs at $\bar{X}$, which are the second nearest neighbors with each other. As a consequence, the line segments $\lambda=\lambda_{p}(\mathbf{k}, \varepsilon)(p=1,2, \ldots, N)$ are divided into two groups, named "a" and "b", each of which forms an infinite continuous line starting from $\lambda=-\infty$ at a pole (at $\mathbf{k}_{3}$ for line "a" and at $\mathbf{k}_{4}$ for line "b") and alternating between $\bar{\Gamma}$ and $\bar{X}$ before diverging to $\lambda=+\infty$. The two lines can intersect one another without interactions since they have opposite mirror parity at all the points of intersection. This, on the other hand, indicates that the behavior of the embedding-potential eigenvalues can qualitatively change when the $\mathbf{k}$-space path connecting the two end points deviates slightly off the mirror symmetry line. To demonstrate this, we show in Figs. 8(c) and 8(d) the embedding-potential eigenvalues of $\mathrm{SnTe}(001)$ and $\mathrm{PbTe}(001)$ along a minor arc (smaller than semicircle) with radius $k_{r}$ connecting the $\bar{\Gamma}$ and $\bar{X}$ points. Here, we have chosen $k_{r}$ as $\sqrt{5}\left|\mathbf{k}_{\bar{X}}\right| / 2$. For PbTe with $n_{M}=0$, the eigenvalue curves in panels (b) and (d) are very similar. On the other hand, for $\mathrm{SnTe}$ with $n_{M}=-2$, in clear contrast to panel (a), two states forming a Kramers pair at $\bar{\Gamma}$ in panel (c) meet again at $\bar{X}$, which is in accord with the $Z_{2}$ topological invariant $v=0$. By comparing Figs. 8(a) and 8(c), we see that the eigenvalues in panel (a) are converted into those in panel (c) in a continuous manner by 

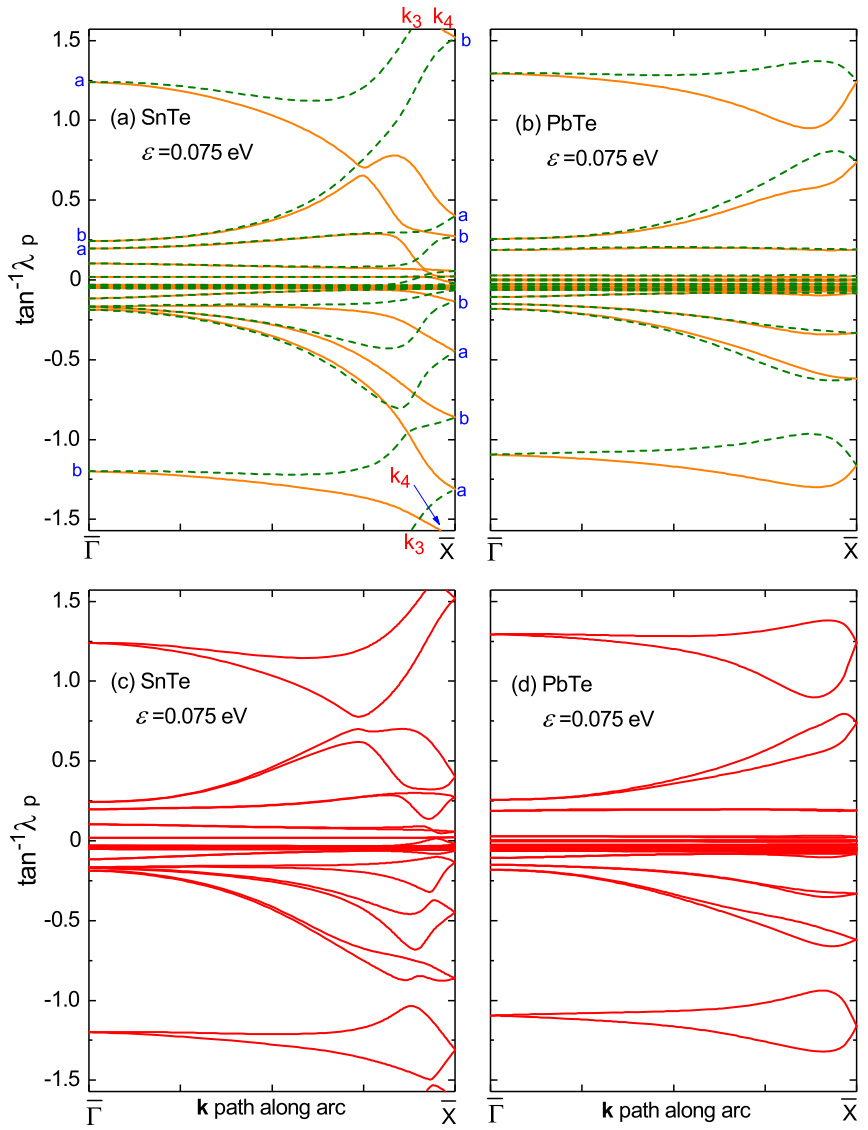

FIG. 8. Upper two panels: Embedding-potential eigenvalues of (a) $\operatorname{SnTe}(001)$ and (b) $\operatorname{PbTe}(001)$ at $\varepsilon=0.075 \mathrm{eV}$ along the $\bar{\Gamma}-\bar{X}$ line. Solid (orange) and dashed (green) lines indicate that corresponding eigenstates $\left|\varphi_{p}\right\rangle$ have mirror parity values, $+i$ and $-i$, about the (110) plane, respectively. (At $-\mathbf{k}$, the eigenvalues are the same as at $+\mathbf{k}$, except that solid (orange) and dashed (green) curves are interchanged.) Lower two panels: Embedding-potential eigenvalues of (c) $\operatorname{SnTe}(001)$ and (d) $\mathrm{PbTe}(001)$ at $\varepsilon=0.075 \mathrm{eV}$ along a k-space minor arc with radius $k_{r}$ connecting two end points, $\bar{\Gamma}$ and $\bar{X}$, where $k_{r}$ is chosen as $k_{r}=\sqrt{5}\left|\mathbf{k}_{\bar{X}}\right| / 2$.

opening a finite " $\lambda$ gap" at every point of intersection between the two lines, "a" and "b", in panel (a).

Returning to Fig. 6(a), it is seen that two surface bands on the bulk truncated SnTe(001) surface intersect $\varepsilon=0.075 \mathrm{eV}$ (red line) along the $\bar{\Gamma}-\bar{X}$ line at $\mathbf{k}_{3}$ and $\mathbf{k}_{4}$. Correspondingly, the embedding-potential eigenvalues of $\operatorname{SnTe}(001)$ at $\varepsilon=$ $0.075 \mathrm{eV}$ in Fig. 8(a) exhibit two poles at $\mathbf{k}_{3}$ and $\mathbf{k}_{4}$. As is seen, the two eigenstates corresponding to the two poles possess opposite mirror parity values. This explains why the two surface bands intersect each other at the Dirac point $\left(\mathbf{k}_{D}, \varepsilon_{D}\right)$ without interactions.

In the case of a TI with $v=1$, a gapless surface band emerges when the embedding-potential eigenvalues switch partners at the two TRIM points as schematically shown in Fig. 2(b). Considering this, it is tempting to argue that two gapless surface bands emerge on $\operatorname{SnTe}(001)$ along the $\bar{\Gamma}-\bar{X}$ line since there are two sets of embedding-potential eigenvalues, "a" and "b", that switch partners at $\bar{\Gamma}$ and $\bar{X}$. While this may be the case for SnTe, we should remember that the degeneracy of
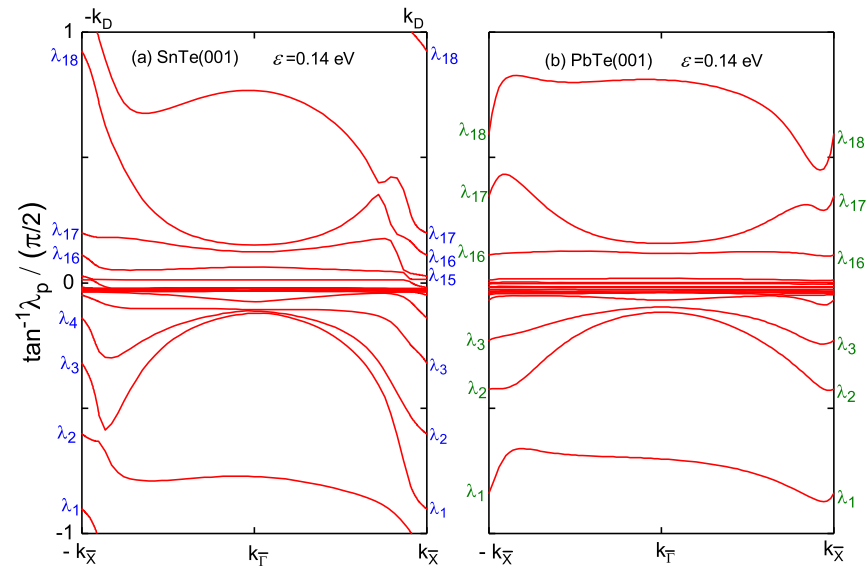

FIG. 9. Embedding-potential eigenvalues $\lambda_{p}(\mathbf{k}, \varepsilon)$ having mirror parity $+i$ about the (110) mirror plane over the whole 1D BZ, $-\mathbf{k}_{\bar{X}} \rightarrow \mathbf{k}_{\bar{\Gamma}} \rightarrow \mathbf{k}_{\bar{X}}$, on a constant energy path $\varepsilon=\varepsilon_{D}(0.14 \mathrm{eV})$. (a) $\operatorname{SnTe}(001)$ and (b) $\mathrm{PbTe}(001)$. The $N / 2=18$ eigenvalues are numbered in ascending order at two end points. $\varepsilon_{D}$ denotes the Dirac-point energy of the surface bands on $\operatorname{SnTe}(001)$ along the $\bar{\Gamma}-\bar{X}$ line shown in Fig. 6(a).

the embedding-potential eigenvalues at $\bar{\Gamma}$ and $\bar{X}$ is a property of time-reversal symmetry rather than mirror symmetry. TCIs with a nonzero mirror Chern number exhibit gapless surface bands even when they do not respect time-reversal symmetry. That is, we should be able to explain the emergence of gapless surface states on the surfaces of TCIs without invoking the degeneracy of the embedding-potential eigenvalues at the TRIM points.

\section{Mirror Chern number}

In order to identify the characteristic feature of the embedding-potential eigenvalues intrinsic to TCIs, we plot in Fig. 9 the $\lambda_{p}(k, \varepsilon)$ values for (a) $\operatorname{SnTe}(001)$ and (b) $\operatorname{PbTe}(001)$ over the whole $1 \mathrm{D} \mathrm{BZ},-\mathbf{k}_{\bar{X}} \rightarrow \mathbf{k}_{\bar{\Gamma}} \rightarrow \mathbf{k}_{\bar{X}}$, on a constant energy path $\varepsilon=\varepsilon_{D}(0.14 \mathrm{eV})$, with the $1 \mathrm{D} k$ axis pointing to the direction from $-\mathbf{k}_{\bar{X}}$ to $\mathbf{k}_{\bar{X}} \cdot\left|\varphi_{p}\right\rangle(p=1,2, \ldots, N)$ along the $\mathbf{k}$-space path are divided into two groups depending on the parity values about the (110) mirror plane, and we plot in Fig. 9 only the $N / 2=18 \lambda_{p}\left(k, \varepsilon_{D}\right)$ values with mirror parity $+i$. (The eigenvalues with mirror parity $-i$ are obtained by replacing $k$ with $-k$, see below.) The two end points, $-\mathbf{k}_{\bar{X}}$ and $\mathbf{k}_{\bar{X}}$, are equivalent, so that all the eigenvalues are the same at the two points. We numbered these eigenvalues, $\lambda_{1}$ through $\lambda_{18}$, in ascending order.

It is seen that the eigenvalue curves for PbTe starting from $\lambda_{p}$ at $-\mathbf{k}_{\bar{X}}$ return to the same value $\lambda_{p}$ at $\mathbf{k}_{\bar{X}}$, so that the eigenvalues form a periodic function over the whole $1 \mathrm{D}$ BZ. On the other hand, the eigenvalue curves for SnTe starting from $\lambda_{p}$ at $-\mathbf{k}_{\bar{X}}$ do not come back to the original value after passing through the 1D BZ. Instead, they are linked to $\lambda_{q}$ with $q=p-2(p \geqslant 3)$ at the end point $\mathbf{k}_{\bar{X}}$. The difference $q-p$ is equal to $n_{+}=-2$, the total Chern number of the valence bands with mirror parity $+i$. As for the two lowest ones with $p=1$ and 2 , the eigenvalue curves starting from $\lambda_{p}$ at $-\mathbf{k}_{\bar{X}}$ diverge to $-\infty$ at a pole, emerge from $+\infty$ after crossing the 

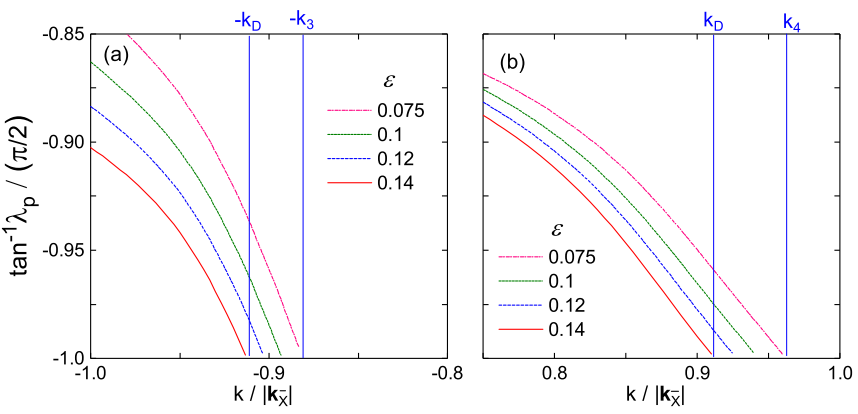

FIG. 10. Magnified view of Fig. 9(a) in bottom region with $\tan ^{-1} \lambda_{p} \leqslant-0.85$ near (a) $k=-\mathbf{k}_{\bar{X}}$ and (b) $k=\mathbf{k}_{\bar{X}}$. In addition to the curve $\lambda=\lambda_{p}(k, \varepsilon)$ at $\varepsilon=0.14 \mathrm{eV}$, corresponding ones at three lower energies $\varepsilon=0.12,0.10$, and $0.075 \mathrm{eV}$ are also plotted.

pole, until they are connected to $\lambda_{q}$ with $q=p-2+N / 2$ at $\mathbf{k}_{\bar{X}}$.

The positions of these poles vary with energy. In Fig. 9(a), where $\varepsilon$ is chosen to be $\varepsilon_{D}=0.14 \mathrm{eV}$, the curve starting from $\lambda_{1}$ exhibits a pole at $-\mathbf{k}_{D}$ before reaching $\lambda_{17}$, whereas the curve starting from $\lambda_{2}$ exhibits a pole at $+\mathbf{k}_{D}$ before reaching $\lambda_{18}$. Since $\frac{\partial}{\partial k} \tan ^{-1} \lambda_{p}<0$ at both poles, according to the discussion in Sec. II G, they should shift in the positive $k$ direction with decreasing energy. In fact, as shown in Fig. 10, when $\varepsilon$ is gradually lowered to $0.075 \mathrm{eV}$ (red line in Fig. 6), the two poles move in the positive $k$ direction and reach $-\mathbf{k}_{3}$ and $+\mathbf{k}_{4}$, respectively. The one-to-one correspondence between the embedding-potential poles and the surface-state energies then indicates that two surface bands with mirror parity $+i$ intersect $\varepsilon=0.14(0.075) \mathrm{eV}$ at $\mathbf{k}_{D}$ and $-\mathbf{k}_{D}\left(\mathbf{k}_{4}\right.$ and $\left.-\mathbf{k}_{3}\right)$ on the bulk truncated $\mathrm{SnTe}(001)$ surface as seen in Fig. 6(a). The slopes of both energy-dispersion curves are negative at these energies.

The Chern number $n_{+}$specifies not only the number of the surface bands with mirror parity $+i$ but also the current direction carried by them. To be more accurate, $n_{+}=-2$ indicates that the number of surface states that intersect $\varepsilon=\varepsilon_{0}$ with $\frac{d \varepsilon_{s}\left(\varepsilon_{0}\right)}{d k}>0$ minus the corresponding one with $\frac{d \varepsilon_{s}\left(\varepsilon_{0}\right)}{d k}<0$ should be $n_{+}=-2$. This is consistent with the behavior of the embedding-potential eigenvalue curves in Fig. 9(a), where the sign of $\frac{\partial}{\partial k} \tan ^{-1} \lambda_{p}$ at the two poles is negative.

The qualitative behavior of the eigenvalues discussed above does not depend on the choice of the energy path. One may consider a curvy path $\varepsilon=\varepsilon_{0}(\mathbf{k})$ satisfying the conditions, $\varepsilon_{v}(\mathbf{k})<\varepsilon_{0}(\mathbf{k})<\varepsilon_{c}(\mathbf{k})$ and $\varepsilon_{0}\left(-\mathbf{k}_{\bar{X}}\right)=\varepsilon_{0}\left(\mathbf{k}_{\bar{X}}\right)$, instead of a constant energy path. In this case also, the eigenvalue curve starting from $\lambda_{1}\left(\lambda_{2}\right)$ at $-\mathbf{k}_{\bar{X}}$ should exhibit at least one pole in order to reach $\lambda_{17}\left(\lambda_{18}\right)$ at $\mathbf{k}_{\bar{X}}$, which indicates that the path $\varepsilon=\varepsilon_{0}(\mathbf{k})$ intersects the energy dispersion curves of surface states with mirror parity $+i$ twice between $-\mathbf{k}_{\bar{X}}$ and $\mathbf{k}_{\bar{X}}$. Since the path $\varepsilon=\varepsilon_{0}(\mathbf{k})$ can be chosen arbitrarily within the projected bulk band gap, there must be two surface bands with mirror parity $+i$ crossing the band gap.

In the present case, the embedding-potential eigenvalues with mirror parity $-i$ are obtained simply by inverting the $\mathbf{k}$ axis in Fig. 9(a) about $\mathbf{k}=0$ thanks to time-reversal symmetry. Then, we will see that the eigenvalue curves with mirror parity $-i$ starting from $\lambda_{p}$ at $-\mathbf{k}_{\bar{X}}$ is linked to $\lambda_{q}$ with $q=p+2(p \leqslant 16)$. The difference $q-p$ is equal to $n_{-}=+2$, the total Chern number of the valence bands with mirror parity $-i$. As for the two highest ones with $p=17$ and 18 , the eigenvalue curves starting from $\lambda_{p}$ at $-\mathbf{k}_{\bar{X}}$ diverge to $+\infty$ at a pole, emerge from $-\infty$ after crossing the pole, until they are connected to $\lambda_{q}$ with $q=p+2-N / 2$ at $\mathbf{k}_{\bar{X}}$. By the same reasoning as above, one can say that there emerge two gapless surface bands with mirror parity $-i$ within the whole BZ. Furthermore, their energy dispersion curves should have a positive velocity $\frac{d \varepsilon_{s}}{d k}>0$ when they intersect a constant energy path $\varepsilon=\varepsilon_{0}$ within the projected bulk band gap.

Putting together all the cases, we have the equation

$$
q-1=\left(p-1+n_{ \pm}\right) \bmod \frac{N}{2}(p, q=1,2, \ldots, N / 2),
$$

which describes how the embedding-potential eigenvalues are connected between two zone boundary points of the whole 1D BZ. One can also express this relationship via a windingnumber-like equation,

$$
n_{ \pm}=\frac{1}{\pi} \sum_{p=1}^{N / 2} \int_{-\mathbf{k}_{\bar{X}}}^{\mathbf{k}_{\bar{X}}} \frac{\partial}{\partial k} \tan ^{-1} \lambda_{p}\left(k, \varepsilon_{0}\right) d k,
$$

where $\varepsilon_{0}$ lies in the projected bulk band gap and the summation is taken over all eigenvalues with either mirror parity $+i$ or $-i$. We infer that the above two equations may hold for other TCIs than SnTe as well, since they ensure the existence of surface bands on a bulk truncated surface that possess properties conforming to a given mirror Chern number with respect to both the number of surface bands and the gradient of their dispersion curves. In part III of the Supplemental Material to this paper [55], we present a short discussion on the relationship between the $Z_{2}$ invariant and the mirror Chern number.

\section{DISCUSSION AND SUMMARY}

As pointed out in the Introduction, several alternative methods are available for determining topological invariants of bulk materials [34-40]. The method based on the flow of the hybrid WCCs is especially efficient since it requires only the calculation of bulk Bloch states $[37,38]$. Compared to this method, the key advantage of the present scheme is that the embedding potential can be used not only for determining topological invariants of a bulk crystal but also for studying the interface between two materials, as will be demonstrated in the Appendix.

The topological phase of a bulk material can also be determined from the flow of surface bands within the band gap. In general, this requires the evaluation of the surface Green's function on a dense mesh of $2 \mathrm{D}(k, \varepsilon)$ points which is computationally more demanding than determining the eigenspectrum of the embedding potential at a single energy. Of course, if one is interested only in the $Z_{2}$ invariant, which is given by the parity of the number of surface bands crossing a line $\varepsilon=\varepsilon_{0}$, one can work at a single energy $\varepsilon_{0}$. However, to determine the Chern number, one must vary the energy value in order to determine the gradient of the dispersion curves of surface bands. In this regard, the present method is computationally advantageous. 
As a final remark, we like to emphasize that computing the surface Green's function $\hat{G}(k, \varepsilon)$ of a semi-infinite system described by a tight-binding Hamiltonian is nowadays quite easy since efficient numerical methods are available [51-54]. Indeed, the surface DOS spectra on a semi-infinite surface of several topological insulators were reported in the past [57-59]. As seen from Eq. (16), the LO based embedding potential is obtained from the surface Green's function just by two matrix multiplications. Hence, the evaluation of this embedding potential is straightforward. It should also be noted that the same quantity is widely used in electron-transport calculations for interfaces [48-50].

In summary, the LO based embedding potential of a semiinfinite crystal is a function of the planar wave vector $\mathbf{k}$ and the one-electron energy $\varepsilon$. It is essentially a bulk quantity that can be constructed from the generalized Bloch states of a bulk tight-binding Hamiltonian. The embedding potential becomes Hermitian when $\varepsilon$ is located within a projected bulk band gap. Its real eigenvalues exhibit distinctly different behavior depending on the topological properties of the valence bands, thus allowing for unambiguous determination of topological invariants of band insulators. To illustrate this, we have presented numerical results for the 2D BHZ Hamiltonian as an example of a 2D time-reversal invariant TI and for SnTe in a rock-salt structure as an example of a 3D TCI with a nonzero mirror Chern number.

\section{ACKNOWLEDGMENTS}

H.I. gratefully acknowledges funding under Japan Society for the Promotion of Science (JSPS) KAKENHI Grant No. JP25110006. H.I. is also grateful to Forschungzentrum Jülich for support during his stay in Jülich.

\section{APPENDIX: INTERFACE BETWEEN BHZ-MODEL SYSTEMS}

To demonstrate that the LO based embedding potential is a useful quantity for studying interface properties, we consider an interface between two semi-infinite TIs represented by the BHZ model. Since the BHZ Hamiltonian is spin diagonal, one can define the spin Chern number, $n_{s}=\left(n_{\uparrow}-n_{\downarrow}\right) / 2$, similar to the mirror Chern number discussed in Sec. IV. In the right-hand-side system $(l \geqslant 1)$, we use the same Hamiltonian parameters as those in Fig. 4(a). By applying Eq. (45) to the $\lambda_{p}$ values at both ends of the 1D Brillouin zone in Fig. 4(a), we see that Chern numbers of both spin components are given by $n_{\uparrow}=+1$ and $n_{\downarrow}=-1$. In the left-hand-side system $(l \leqslant 0)$, $\varepsilon_{1}$ and $\varepsilon_{2}$ are changed to -0.75 and 0.75 , respectively. In addition, the sign of the hopping integral $t_{12}$ in the $x$ direction [see Fig. 3(a)] is reversed in order to reverse the sign of the spin Chern number of the left-hand-side system. Figures 11(a) and 11(b) show the surface DOS spectra of both systems for the up-spin component when two systems are separated. Both surfaces exhibit a metallic surface band with $d \varepsilon_{s} / d k_{x}>0$,
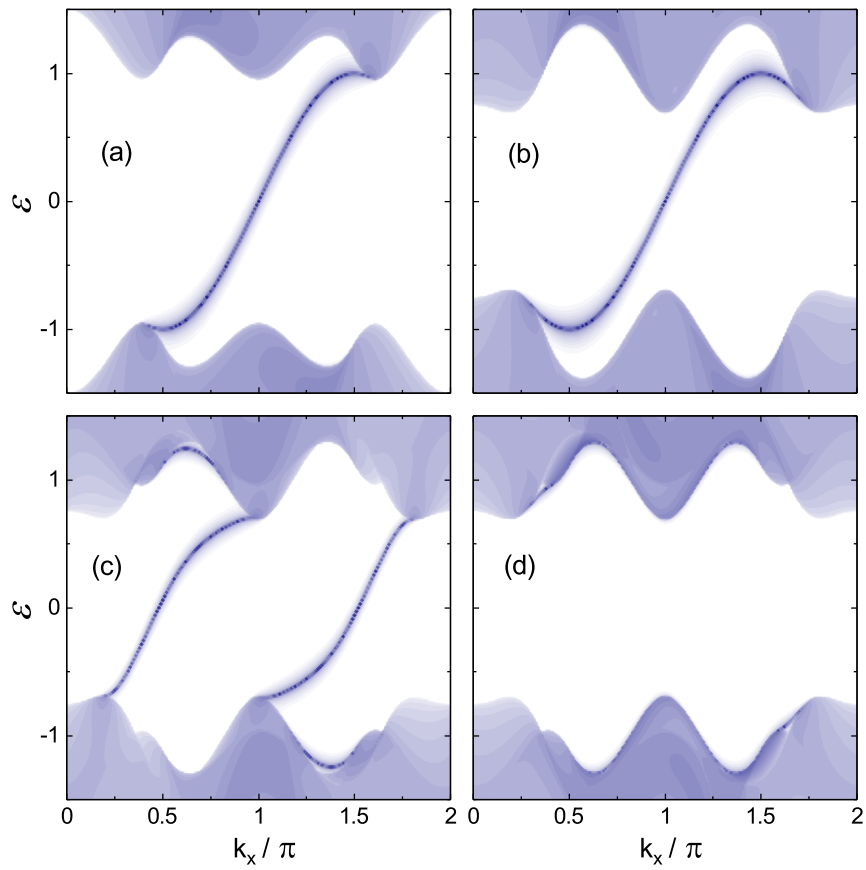

FIG. 11. (a) Surface DOS of up-spin electrons for the right-handside system, $\rho_{1}\left(k_{x}, \varepsilon\right)$, and (b) that for the left-hand-side system, $\rho_{0}\left(k_{x}, \varepsilon\right)$, when both systems with opposite spin Chern numbers are decoupled. (c) and (d): DOS spectra of up-spin electrons at the interface layer $(l=1)$ when both systems are fully coupled. In panel (c), both systems have opposite spin Chern numbers, while they share the same spin Chern number in panel (d).

whose sign is consistent with the $n_{\uparrow}$ values of both systems. Here, it should be noted that the sign of $d \varepsilon_{s} / d k_{x}$ is reversed between the top and bottom surfaces of a crystal.

Now, we couple both systems by switching on the interlayer hopping terms, which are assumed to be the same as in the bulk. The DOS spectrum at the interface can be calculated easily from the Green's function of the interface layer $(l=1)$,

$$
\hat{G}_{11}\left(k_{x}, \varepsilon\right)=\left[\varepsilon \hat{I}-\hat{H}_{11}^{k_{x}}-\hat{\Sigma}_{L}-\hat{\Sigma}_{R}\right]^{-1},
$$

where $\hat{\Sigma}_{L}\left(\hat{\Sigma}_{R}\right)$ denotes the LO based embedding potential of the semi-infinite system on the left-hand (right-hand) side. Figure 11(c) shows the DOS spectrum of up-spin electrons at the interface layer. Since both systems possess opposite spin Chern numbers, metallic states with topological origin appear at the interface. The surface bands of both systems shown in Figs. 11(a) and 11(b) have the same spin. Thus, they interact and split, resulting in the two interface bands shown in panel (c). Now, we reverse the sign of the spin Chern number of the left-hand-side system again by reversing the sign of $t_{12}$ in the $x$ direction. Figure 11(d) shows the resultant DOS spectrum of up-spin electrons at the interface. Since both systems are now in the same topological phase, the metallic edge states at the interface disappear.
[1] M. Z. Hasan and C. L. Kane, Rev. Mod. Phys. 82, 3045 (2010).

[2] X. L. Qi and S. C. Zhang, Rev. Mod. Phys. 83, 1057 (2011).
[3] A. Bansil, H. Lin, and T. Das, Rev. Mod. Phys. 88, 021004 (2016). 
[4] C. K. Chiu, J. C. Y. Teo, A. P. Schnyder, and S. Ryu, Rev. Mod. Phys. 88, 035005 (2016).

[5] C. L. Kane and E. J. Mele, Phys. Rev. Lett. 95, 146802 (2005).

[6] C. L. Kane and E. J. Mele, Phys. Rev. Lett. 95, 226801 (2005).

[7] L. Fu and C. L. Kane, Phys. Rev. B 74, 195312 (2006).

[8] J. E. Moore and L. Balents, Phys. Rev. B 75, 121306(R) (2007).

[9] B. A. Bernevig, T. L. Hughes, and S. C. Zhang, Science 314, 1757 (2006).

[10] M. König, S. Wiedmann, C. Brüne, A. Roth, H. Buhmann, L. W. Molenkamp, X. L. Qi, and S. C. Zhang, Science 318, 766 (2007).

[11] S. Murakami, Phys. Rev. Lett. 97, 236805 (2006).

[12] M. Wada, S. Murakami, F. Freimuth, and G. Bihlmayer, Phys. Rev. B 83, 121310(R) (2011).

[13] L. Fu, C. L. Kane, and E. J. Mele, Phys. Rev. Lett. 98, 106803 (2007).

[14] J. C. Y. Teo, L. Fu, and C. L. Kane, Phys. Rev. B 78, 045426 (2008).

[15] H. Zhang, C. X. Liu, X. L. Qi, X. Dai, Z. Fang, and S. C. Zhang, Nat. Phys. 5, 438 (2009).

[16] Y. Xia, D. Qian, D. Hsieh, L. Wray, A. Pal, H. Lin, A. Bansil, D. Grauer, Y. S. Hor, R. J. Cava, and M. Z. Hasan, Nat. Phys. 5, 398 (2009).

[17] K. Kuroda, M. Arita, K. Miyamoto, M. Ye, J. Jiang, A. Kimura, E. E. Krasovskii, E. V. Chulkov, H. Iwasawa, T. Okuda, K. Shimada, Y. Ueda, H. Namatame, and M. Taniguchi, Phys. Rev. Lett. 105, 076802 (2010).

[18] B. Yan, C. X. Liu, H. J. Zhang, C. Y. Yam, X. L. Qi, T. Frauenheim, and S. C. Zhang, Europhys. Lett. 90, 37002 (2010).

[19] H. Lin, R. S. Markiewicz, L. A. Wray, L. Fu, M. Z. Hasan, and A. Bansil, Phys. Rev. Lett. 105, 036404 (2010).

[20] L. Fu, Phys. Rev. Lett. 106, 106802 (2011).

[21] T. H. Hsieh, H. Lin, J. Liu, W. Duan, A. Bansil, and L. Fu, Nat. Commun. 3, 982 (2012).

[22] S. Y. Xu, C. Liu, N. Alidoust, M. Neupane, D. Qian, I. Belopolski, J. D. Denlinger, Y. J. Wang, H. Lin, L. A. Wray, G. Landolt, B. Slomski, J. H. Dil, A. Marcinkova, E. Morosan, Q. Gibson, R. Sankar, F. C. Chou, R. J. Cava, A. Bansil, and M. Z. Hasan, Nat. Commun. 3, 1192 (2012).

[23] Y. Tanaka, Z. Ren, T. Sato, K. Nakayama, S. Souma, T. Takahashi, K. Segawa, and Y. Ando, Nat. Phys. 8, 800 (2012).

[24] P. Dziawa, B. J. Kowalski, K. Dybko, R. Buczko, A. Szczerbakow, M. Szot, E. Łusakowska, T. Balasubramanian, B. M. Wojek, M. H. Berntsen, O. Tjernberg, and T. Story, Nat. Mater. 11, 1023 (2012).

[25] E. O. Wrasse and T. M. Schmidt, Nano Lett. 14, 5717 (2014).

[26] K. Kobayashi, Surf. Sci. 639, 54 (2015).

[27] C. Niu, P. M. Buhl, G. Bihlmayer, D. Wortmann, S. Blügel, and Y. Mokrousov, Nano Lett. 15, 6071 (2015).

[28] C. Niu, P. M. Buhl, G. Bihlmayer, D. Wortmann, S. Blügel, and Y. Mokrousov, 2D Mater. 3, 025037 (2016).

[29] Y. Hatsugai, Phys. Rev. Lett. 71, 3697 (1993).
[30] G. Graf and M. Porta, Commun. Math. Phys. 324, 851 (2013).

[31] X. Dang, J. D. Burton, A. Kalitsov, J. P. Velev, and E. Y. Tsymbal, Phys. Rev. B 90, 155307 (2014).

[32] J. Betancourt, S. Li, X. Dang, J. D. Burton, E. Y. Tsymbal, and J. P. Velev, J. Phys.: Condens. Matter 28, 395501 (2016).

[33] L. Fu and C. L. Kane, Phys. Rev. B 76, 045302 (2007).

[34] T. Fukui and Y. Hatsugai, J. Phys. Soc. Jpn. 76, 053702 (2007).

[35] A. A. Soluyanov and D. Vanderbilt, Phys. Rev. B 83, 235401 (2011).

[36] R. Yu, X. L. Qi, A. Bernevig, Z. Fang, and X. Dai, Phys. Rev. B 84, 075119 (2011).

[37] M. Taherinejad, K. F. Garrity, and D. Vanderbilt, Phys. Rev. B 89, 115102 (2014).

[38] D. Gresch, G. Autès, O. V. Yazyev, M. Troyer, D. Vanderbilt, B. A. Bernevig, and A. A. Soluyanov, Phys. Rev. B 95, 075146 (2017).

[39] A. M. Turner, Y. Zhang, and A. Vishwanath, Phys. Rev. B 82, 241102(R) (2010).

[40] T. L. Hughes, E. Prodan, and B. A. Bernevig, Phys. Rev. B 83, 245132 (2011).

[41] H. Ishida and D. Wortmann, Phys. Rev. B 93, 115415 (2016).

[42] J. E. Inglesfield, J. Phys. C 14, 3795 (1981).

[43] J. E. Inglesfield, The Embedding Methodfor Electronic Structure (IOP publishing, Bristol, 2015).

[44] D. Wortmann, H. Ishida, and S. Blügel, Phys. Rev. B 65, 165103 (2002).

[45] D. Wortmann, H. Ishida, and S. Blügel, Phys. Rev. B 66, 075113 (2002).

[46] M. James and S. Crampin, Phys. Rev. B 81, 155439 (2010).

[47] N. Marzari, A. A. Mostofi, J. R. Yates, I. Souza, and D. Vanderbilt, Rev. Mod. Phys. 84, 1419 (2012).

[48] M. Brandbyge, J. L. Mozos, P. Ordejón, J. Taylor, and K. Stokbro, Phys. Rev. B 65, 165401 (2002).

[49] Y. Xue and M. A. Ratner, Phys. Rev. B 68, 115406 (2003).

[50] K. S. Thygesen and A. Rubio, Phys. Rev. B 77, 115333 (2008).

[51] S. Sanvito, C. J. Lambert, J. H. Jefferson, and A. M. Bratkovsky, Phys. Rev. B 59, 11936 (1999).

[52] J. Taylor, H. Guo, and J. Wang, Phys. Rev. B 63, 245407 (2001).

[53] I. Rungger and S. Sanvito, Phys. Rev. B 78, 035407 (2008).

[54] M. P. L. Sancho, J. M. L. Sancho, J. M. L. Sancho, and J. Rubio, J. Phys. F 15, 851 (1985).

[55] See Supplemental Material at http://link.aps.org/supplemental/ 10.1103/PhysRevB.96.125413 for results of additional calculations for the BHZ model and a short discussion on topological invariants.

[56] C. S. Lent, M. A. Brown, J. D. Dow, and R. S. Allgaier, Superlattices Microstruct. 2, 491 (1986).

[57] H. J. Zhang, C. X. Liu, X. L. Qi, X. Y. Deng, X. Dai, S. C. Zhang, and Z. Fang, Phys. Rev. B 80, 085307 (2009).

[58] A. Narayan, I. Rungger, and S. Sanvito, Phys. Rev. B 86, 201402(R) (2012).

[59] Y. Shi, M. Wu, F. Zhang, and J. Feng, Phys. Rev. B 90, 235114 (2014). 